\title{
ENLARGEMENTS OF REGULAR SEMIGROUPS
}

\author{
by M. V. LAWSON
}

(Received 30th May 1994)

\begin{abstract}
We introduce a class of regular extensions of regular semigroups, called enlargements; a regular semigroup $T$ is said to be an enlargement of a regular subsemigroup $S$ if $S=S T S$ and $T=T S T$. We show that $S$ and $T$ have many properties in common, and that enlargements may be used to analyse a number of questions in regular semigroup theory.
\end{abstract}

1991 Mathematics subject classification 20M17.

\section{Introduction}

Quasi-ideals were introduced by Otto Steinfeld [43] as those non-empty subsets $Q$ of a semigroup $T$ satisfying $Q T \cap T Q \subseteq Q$. When $T$ is regular they are precisely the subsets $Q$ of $T$ which satisfy $Q T Q=Q([43$, Theorem 9.3]). There are many examples of quasi-ideals in regular semigroup theory. We list below some of the most important:

- Every subsemigroup of the form $e S e$ (where $e$ is an idempotent) is a quasi-ideal of $S$. Such subsemigroups are called local submonoids.

- McAlister [30] studies quasi-ideal embeddings of one regular semigroup in another.

- Quasi-ideal embeddings arise naturally in the inverse case. Let $\Sigma$ be a structure whose semigroup of partial automorphisms $\Gamma(\Sigma)$ is an inverse semigroup. Let $\Sigma$ be embedded in another structure $\Sigma^{\prime}$ of the same type such that the semigroup $\Gamma\left(\Sigma^{\prime}\right)$ of partial automorphisms is also an inverse semigroup. Then $\Gamma(\Sigma)$ is a quasi-ideal of $\Gamma\left(\Sigma^{\prime}\right)$.

- In [24] and [29], McAlister shows that every inverse semigroup can be embedded as a quasi-ideal in a factorisable inverse semigroup.

If $S$ is a regular subsemigroup and quasi-ideal of the regular semigroup $T$ (to be brief, we call $S$ a regular quasi-ideal of $T$ ), then the semigroups $S$ and $T$ may still have little in common. Consider now the subset $T^{\prime}=T S T$. It can be shown that $T^{\prime}$ is a regular subsemigroup of $T$ containing $S$. But it is also easily verified that both $S=S T^{\prime} S$ and $T^{\prime}=T^{\prime} S T^{\prime}$. Thus $S$ is embedded as a quasi-ideal of $T^{\prime}$ possessing the additional property that $T^{\prime}=T^{\prime} S T^{\prime}$. We call such embeddings enlargements, and it is the thesis of this paper that enlargements provide a useful new idea in the study of regular semigroups. Two examples may serve to provide some partial support to this contention. 
Example 1. It can be shown that a regular semigroup $T$ is an enlargement of a subsemigroup of the form $e T e$, where $e$ is an idempotent, precisely when $T=T e T$. Semigroups possessing such idempotents have been considered by a number of authors.

Example 2. In [18], we showed that an E-unitary inverse semigroup is over a semilattice (in the sense of McAlister [25]) if, and only if, it has an enlargement which is a semidirect product of a group and a semilattice. On the basis of this result, we proved in [18] that every inverse semigroup has an enlargement which is close to being factorisable (what we call almost factorisable), sharpening the results of [24] and [29].

These examples suggested to us that enlargements be investigated in the broader frame of arbitrary regular semigroups. Further motivation comes from recent work of Talwar [44] (and private communication) on the Morita theory of semigroups; it can be shown that if $T$ is an enlargement of $S$ then $T$ and $S$ are Morita equivalent.

The main aim of this paper is to uncover properties a semigroup and its enlargements have in common and to provide examples and applications of this idea in regular semigroup theory.

The paper is divided into eight short sections. Section 1 is a preliminary section in which the results from regular semigroup theory needed to understand this paper are presented. In Section 2, we provide an alternative characterisation of enlargements and give some examples of enlargements in regular semigroup theory. In Section 3, we study the relationship between the ideal structure of a semigroup $S$ and an enlargement $T$. We prove that $S$ and $T$ have isomorphic lattices of two-sided ideals and isomorphic posets of principal ideals (Theorem 3.3). We also prove that $S$ is combinatorial (resp. bisimple, simple) precisely when $T$ is combinatorial (resp. bisimple, simple). In Section 4, we generalise McAlister's Local Structure Theorem [26], and prove in Corollary 4.3 that $T$ is a locally isomorphic image of a regular Rees matrix semigroup over $S$. In Section 5, we show that $S$ and $T$ have isomorphic lattices of idempotent separating congruences (Theorem 5.4). In Section 6, we specialise to the case where both $S$ and $T$ are inverse semigroups. We prove in Theorem 6.4, that the lattices of all congruences of $S$ and $T$ are isomorphic. In Theorems 6.11 and 6.12, we show how to construct all inverse enlargements of an inverse monoid by means of Rees matrix semigroups. In Section 7, we show how the notion of enlargement may be defined for ordered groupoids. We prove in Proposition 7.2, that $T$ is an enlargement of $S$ if, and only if, the ordered groupoid $\mathbf{G}(T)$ is an enlargement of the ordered groupoid $\mathbf{G}(S)$, where $\mathbf{G}(U)$ is the ordered groupoid constructed from the regular semigroup $U$ by Nambooripad [36]. At the conclusion of this section, we explain how our definition of enlargement arose from work of Ehresmann and McAlister. In the final section, Section 8, we discuss a number of applications of enlargements to regular semigroup theory.

Although enlargements can be defined for non-regular semigroups, we shall only consider in this paper regular semigroups and their regular subsemigroups, consequently, enlargements will always be by regular semigroups of regular subsemigroups. 


\section{Preliminaries}

In this section, we review the results from regular semigroup theory needed to follow this paper.

As usual we denote Green's relations by $\mathscr{L}, \mathscr{R}, \mathscr{H}, \mathscr{D}$, and $\mathscr{J}$. If $S$ is a subsemigroup of $T$ and $\mathscr{K}$ is one of Green's relations on $S$ then we shall often denote it by $\mathscr{K}(S)$ to avoid ambiguity. Similarly, if $\rho$ is a relation defined on $S$ we shall often emphasise this fact by writing $\rho(S)$.

We denote the set of idempotents of a semigroup $S$ by $E(S)$. When $A$ is a subset of $S$ then $E(A)=A \cap E(S)$. A regular semigroup is said to be orthodox if $E(S)$ is a subsemigroup. In the case where $E(S)=S$, then $S$ is called a band. If $x$ is a regular element then $V(x)$ denotes the set of all inverses of $x$. If $x^{\prime} \in V(x)$ then, as usual, $x=x x^{\prime} x$ and $x^{\prime}=x^{\prime} x x^{\prime}$. A pair of elements $\left(x, x^{\prime}\right)$ in a regular semigroup $S$ is called an inverse pair if $x^{\prime} \in V(x)$. by:

If $e$ and $f$ are idempotents then the sandwich set of $e$ and $f$, denoted $S(e, f)$, is defined

$$
S(e, f)=\{h \in E(S): f h e=h \quad \text { and } \quad e h f=e f\}
$$

In a regular semigroup the sandwich sets are always non-empty [36]. It is well-known and easy to check that $h \in S(e, f)$ if, and only if, $h \in E(S) \cap f V(e f) e$.

We have already recalled that a non-empty subset $Q$ of a semigroup $S$ is called a quasi-ideal of $S$ if $Q S \cap S Q \subseteq Q$. The collection of all quasi-ideals of $S$ is denoted by $\mathscr{2}(S)$. It can be shown that a non-empty subset $Q$ of $S$ is a quasi-ideal if, and only if, it is the intersecton of a left ideal and a right ideal of $S$ ([43, Corollary 2.7]). A quasiideal $Q$ is said to be regular if it is a regular subsemigroup. The following result ([43, Theorem 9.3]) provides some useful characterisations of quasi-ideals in the case where $S$ is regular.

Result 1.1. The following conditions on a semigroup $S$ are equivalent:

(i) $S$ is regular.

(ii) For every right ideal $R$ and left ideal $L$ of $S$, we have that $R L=R \cap L$.

(iii) $\mathscr{Q}(S)$ is a regular semigroup with respect to multiplication of subsets of $S$.

(iv) Every quasi-ideal $Q$ of $S$ has the form $Q=Q S Q$.

A useful tool in our work is the natural partial order definable on any regular semigroup. Let $S$ be a regular semigroup. Define $x \leq y$ if, and only if, $R_{x} \leq R_{y}$ and $x=f y$ some $f \in E\left(R_{x}\right)$. If $e$ and $f$ are idempotents then $e \leq f$ iff $e=e f=f e$, the usual ordering on idempotents. When regular semigroups are considered as posets it will always be with respect to their natural partial order. The following is Proposition 1.2 of [37].

Result 1.2. Let $x$ and $y$ belong to the regular semigroup $S$. Then the following are equivalent.

(i) $x \leq y$.

(ii) For every $f \in E\left(R_{y}\right)$ there exists $e \in E\left(R_{x}\right)$ such that $e \leq f$ and $x=e y$. 
(iii) For every $f \in E\left(L_{y}\right)$ there exists $e \in E\left(L_{x}\right)$ such that $e \leq f$ and $x=y e$.

(iv) $L_{x} \leq L_{y}$ and $x=y f$ for some $f \in E\left(L_{x}\right)$.

If $(P, \leq)$ is a poset then a subset $Q$ of $P$ is said to be an order ideal if $p \leq q \in Q$ implies that $p \in Q$. The subset $[q]=\{p: p \in P$ and $p \leq q\}$ is called a principal order ideal of $P$. The following result shows that to check whether a regular subsemigroup is an order ideal it is enough to concentrate on the idempotents.

Lemma 1.3. Let $S$ be a regular subsemigroup of a regular semigroup $T$. Then $E(S)$ is an order ideal of $E(T)$ if, and only if, $S$ is an order ideal of $T$.

Proof. Let $E(S)$ be an order ideal of $E(T)$ and suppose that $x \leq y \in S$. Then by Result 1.2(ii), if $f \in E\left(R_{y}\right) \cap S$ then there exists $e \in E\left(R_{x}\right)$ such that $e \leq f$ and $x=e y$. By assumption, $e \in S$ and so $x \in S$. The converse is clear.

The following was first proved in [35] (I am grateful to the referee for pointing this out to me).

Result 1.4. Let $S$ be any semigroup and let $e$ and $f$ be any $\mathscr{D}$-related idempotents of S. Then eSe and fSf are isomorphic..

The properties of a regular semigroup are often closely related to the properties of its local submonoids. If $\mathscr{P}$ is a property of regular semigroups then a semigroup $S$ is said to be locally $\mathscr{P}$ if all the local submonoids of $S$ have property $\mathscr{P}$. An example of the importance of the properties of local submonoids concerns the natural partial order. Although for the class of inverse semigroups (those regular semigroups in which each element has a unique inverse) the natural partial order is compatible with the multiplication, this is not true of all regular semigroups. The general situation is described by the following result [37].

Result 1.5. In a regular semigroup $S$, the natural partial order is compatible with the multiplication if, and only if $S$ is locally inverse.

An orthodox regular semigroup which is also locally inverse is said to be a generalised inverse semigroup. It can be shown that a band is locally inverse if, and only if, it is normal (see Chapter IV, Exercise 12 of [13]). It follows that the generalised inverse semigroups are just the orthodox semigroups with a normal band of idempotents.

Let $S$ be a regular semigroup. The trace product of $x$ and $y$, denoted $x \cdot y$, is defined to be $x y$ if $x y \in R_{x} \cap L_{y}$ and undefined otherwise. In the result below, (i) is Theorem 3 of [34] combined with Theorem II.3.5 of [13], (ii) is Theorem 1.2 of [36], (iii) is proved by induction, and the proof of (iv) is Theorem 1.6 of [37].

Result 1.6. Let $S$ be a regular semigroup.

(i) $x \cdot y$ exists if, and only if, there is $x^{\prime} \in V(x)$ and $y^{\prime} \in V(y)$ such that $x^{\prime} x=y y^{\prime}$. 
(ii) Let $x, y \in S, x^{\prime} \in V(x), y^{\prime} \in V(y)$ and $h \in S\left(x^{\prime} x, y y^{\prime}\right)$. Then

$$
x y=(x h) \cdot(h y) \text { and } y^{\prime} h x^{\prime} \in V(x y) .
$$

(iii) Let $x_{1}, \ldots, x_{n} \in S, x_{1}^{\prime} \in V\left(x_{1}\right)$ and $x_{n}^{\prime} \in V\left(x_{n}\right)$. Then

$$
V\left(x_{1} \ldots x_{n}\right) \cap x_{n}^{\prime} S x_{1}^{\prime} \neq \varnothing \text {. }
$$

(iv) Let $x=x_{1} \ldots x_{n}$, where $x_{1}, \ldots, x_{n} \in S$. Then there exist elements $y_{1}, \ldots, y_{n} \in S$ such that $y_{i} \leq x_{i}$ and $x=y_{1} \ldots y_{n}$ is a trace product.

A useful result is the following, which is Lemma 2.2 of [37].

Result 1.7. Let $S$ be a regular semigroup.

(i) If $y \mathscr{D} y^{\prime}$ then for every $x \leq y$ there exists $x^{\prime} \mathscr{D} x$ such that $x^{\prime} \leq y^{\prime}$.

(ii) $S x S \subseteq S y S$ if, and only if, there exists $y^{\prime}$ such that $x \mathscr{D} y^{\prime} \leq y$.

Let $S$ be a regular semigroup. Then the intersection of all congruences $\rho$ on $S$ such that $S / \rho$ is inverse is a congruence, denoted by $\gamma$, such that $S / \gamma$ is inverse. The congruence $\gamma$ is called the minimum inverse semigroup congruence. The following is proved as Theorem VI.1.12 of [13].

Result 1.8. Let $S$ be an orthodox semigroup. Then the following three conditions are equivalent:

(i) $(x, y) \in \gamma$.

(ii) $V(x)=V(y)$.

(iii) $V(x) \cap V(y)$ is non-empty.

A homomorphism $\theta: S \rightarrow T$ is said to be a local isomorphism if the restrictions $(\theta \mid e S e)$ are injective for all $e \in E(S)$. The following was proved as Lemma 1.3 of [27].

Result 1.9. Let $\theta: S \rightarrow T$ be a local isomorphism between regular semigroups. Then for all $x, y \in S$, the restrictions $(\theta \mid x S y)$ are injective.

The following result is fundamental to our work on inverse enlargements of inverse monoids. It was proved as Proposition 1.4 of [27]. As usual, $\gamma^{\natural}$ denotes the natural map associated with the congruence $\gamma$.

Result 1.10. Let $S$ be a regular semigroup. Then $\gamma^{\natural}: S \rightarrow S / \gamma$ is a local isomorphism if, and only if, $S$ is a generalised inverse semigroup.

We now recall the definition and properties of Rees matrix semigroups. Let $S$ be a semigroup, and let $I$ and $\Lambda$ be sets. Let $p: \Lambda \times I \rightarrow S$ be a function; we write 
$p(\lambda, i)=p_{\lambda, i}$. Put $M=M(S, I, \Lambda, p)=I \times S \times \Lambda$ equipped with the multiplication given by

$$
(i, s, \lambda)(j, t, u)=\left(i, s p_{\lambda . j} t, \mu\right) .
$$

Then $M$ is called a Rees matrix semigroup over $S$. The function $p$ is called the sandwich function. Put $R=R M(S, I, \Lambda, p)$ the set of all regular elements of $M$. It was proved in [26] that $R$ is a regular semigroup if $S$ is a regular semigroup. The following is proved in [26].

Result 1.11. Let $S$ be an inverse semigroup. Then for any sandwich function $R M(S, I, \Lambda, p)$ is a regular, locally inverse semigroup.

If $S$ has a zero, 0 , then the set $I=\{(i, 0, \lambda): i \in I, \lambda \in \Lambda\}$ is an ideal of $M(S, I, \Lambda, P)$. The Rees quotient $M(S, I, \Lambda, P) / I$ is denoted by $M^{\circ}(S, I, \Lambda, P)$.

Finally, define a relation $\rho$ on any regular semigroup $S$ by

$$
(a, b) \in \rho \text { if, and only if, } x a y=x b y \text { for all } x, y \in S .
$$

Then we have the following result from [28].

Result 1.12. $\rho$ is the largest congruence on $S$ with the property that $\rho^{\natural}: S \rightarrow S / \rho$ is a local isomorphism.

For any remaining undefined terms from semigroup theory consult Howie [13] and Petrich [39].

\section{Basic properties of enlargements}

We have defined a regular semigroup $T$ to be an enlargement of a regular subsemigroup $S$ if $S T S=S$ and $T S T=T$. This is equivalent to saying that $S \in V(T)$ in the semigroup $2(T)$ of all quasi-ideals of $T$. More generally, we say that a regular semigroup $T$ is an enlargement of a regular semigroup $S$ if there is an embedding $\imath: S \rightarrow T$ such that $T$ is an enlargement of $\imath(S)$.

Our first result provides a useful alternative characterisation of enlargements. Let $S$ be a regular subsemigroup of a regular semigroup $T$. We shall refer to the following three properties:

(E1) $E(S)$ is an order ideal of $E(T)$.

(E2) If $x \in T$ and for some $x^{\prime} \in V(x), x^{\prime} x, x x^{\prime} \in S$ then $x \in S$.

(E3) For each $e \in E(T)$ there exists $f \in E(S)$ such that $e \mathscr{D} f$.

Theorem 2.1. Let $S$ be a regular subsemigroup of a regular semigroup $T$.

(i) $S$ is a quasi-ideal of $T$ if, and only if. (E1) and (E2) hold.

(ii) $T$ is an enlargement of $S$ if, and only if, $S$ is a quasi-ideal of $T$ and (E3) holds. 
Proof. (i) Let $S$ be a quasi-ideal of $T$. (E1) holds: let $f \leq e \in E(S)$. Then $f=e f e \in S T S=S$. Thus $f \in E(S)$. (E2) holds: let $x \in T$ and $x^{\prime} \in V(x)$ be such that $x^{\prime} x, x x^{\prime} \in S$. Then $x=x x^{\prime} . x \cdot x^{\prime} x \in S T S=S$.

To prove the converse, suppose that (E1) and (E2) hold. Let $u v w \in S T S$ where $u, w \in S$. Choose $u^{\prime} \in V(u) \cap S$ and $w^{\prime} \in V(w) \cap S$. Then by Result 1.6(iii), there exists $z \in V(u v w) \cap w^{\prime} T u^{\prime}$. Thus $z=w^{\prime} t u^{\prime}$ for some $t \in T$. It is clear that

$$
(u v w) z \leq u u^{\prime} \text { and } z(u v w) \leq w^{\prime} w .
$$

By (E1), $(u v w) z, z(u v w) \in S$. Thus by (E2), we have that $u v w \in S$.

(ii) Suppose that $S$ is a quasi-ideal of $T$ (in fact, it is enough to assume that $S$ is an order ideal of $T$ ), and that $T=T S T$. We show first that each element $t \in T$ can be written as a trace product $t=u \cdot w \cdot v$ where $u, v \in T$ and $w \in S$. Clearly $t=a c b$ where $a, b \in T$ and $c \in S$. By Result 1.6(iv), there exist elements $u, v, w$ such that $u \leq a, v \leq b$ and $w \leq c$ and $t=u \cdot w \cdot v$ is a trace product. By (i), $S$ a quasi-ideal implies that (E1) holds and so by Lemma $1.3, S$ is an order ideal of $T$. Thus $w \in S$. Now we show that (E3) holds. Let $e \in E(T)$. Then we can write $e=u \cdot w \cdot v$, a trace product with $w \in S$. In particular, $e \mathscr{D} w$. Choose $w^{\prime} \in V(w) \cap S$. Then $e \mathscr{D} w^{\prime} w \in E(S)$.

Conversely, let $S$ be a quasi-ideal of $T$ and suppose that (E3) holds. We show that $T \subseteq T S T$. Let $t \in T$ and $t^{\prime} \in V(t)$. Then by (E3) there are idempotents $e, f \in E(S)$ such that $t^{\prime} t \mathscr{D} e$ and $t t^{\prime} \mathscr{D} f$. By Proposition II.3.6 of [13], we can pick elements $a, b \in T$ and inverses $a^{\prime} \in V(a)$ and $b^{\prime} \in V(b)$ such that

$$
a a^{\prime}=t^{\prime} t, \quad a^{\prime} a=e, \quad b b^{\prime}=t t^{\prime} \quad \text { and } \quad b^{\prime} b=f .
$$

Put $s=b^{\prime} t a$. Then $f s e=\left(b^{\prime} b\right) b^{\prime} t a\left(a^{\prime} a\right)=s$. Thus $s=f s e \in S T S \subseteq S$. But $b s a^{\prime}=$ $\left(b b^{\prime}\right) t\left(a a^{\prime}\right)=\left(t t^{\prime}\right) t\left(t^{\prime} t\right)=t$. Thus $t \in T S T$ as required.

The idea used to prove the last part of Theorem 2.1(ii) is the basis of an important decomposition technique for inverse pairs.

Lemma 2.2. Let $T$ be an enlargement of $S$. Then for every inverse pair $\left(t, t^{\prime}\right)$ in $T$ there exist inverse pairs $\left(x, x^{\prime}\right)$ and $\left(y, y^{\prime}\right)$ in $T$ and an inverse pair $\left(s, s^{\prime}\right)$ in $S$ such that

$$
t=x \cdot s \cdot y^{\prime}, x x^{\prime}=t t^{\prime}, y y^{\prime}=t^{\prime} t, x^{\prime} x=s s^{\prime} \quad \text { and } \quad y^{\prime} y=s^{\prime} s .
$$

Proof. By (E3), there exist idempotents $e, f \in E(S)$, such that $t t^{\prime} \mathscr{D} e$ and $t^{\prime} t \mathscr{D} f$. By proposition II.3.6 of [13], there exist $x, y \in T, x^{\prime} \in V(x)$ and $y^{\prime} \in V(y)$ such that $t t^{\prime}=x x^{\prime}, e=x^{\prime} x, t^{\prime} t=y y^{\prime}$, and $f=y^{\prime} y$. Put $s=x^{\prime} t y$ and $y^{\prime} t^{\prime} x$. Then

$$
s=x^{\prime} t y=\left(x^{\prime} x\right)\left(x^{\prime} t y\right)\left(y^{\prime} y\right)=e\left(x^{\prime} t y\right) f \in S T S=S,
$$

and

$$
s^{\prime}=y^{\prime} t^{\prime} x=\left(y^{\prime} y\right)\left(y^{\prime} t^{\prime} x\right)\left(x^{\prime} x\right)=f\left(y^{\prime} t^{\prime} x\right) e \in S T S=S,
$$


since $S$ is a quasi-ideal of $T$. Now,

$$
s s^{\prime}=\left(x^{\prime} t y\right)\left(y^{\prime} t^{\prime} x\right)=x^{\prime} x ; \quad \text { and } \quad s^{\prime} s=y^{\prime} y
$$

and

$$
s s^{\prime} s=x^{\prime} x\left(x^{\prime} t y\right)=x^{\prime} t y=s \quad \text { and } \quad s^{\prime} s s^{\prime}=y^{\prime} y\left(y^{\prime} t^{\prime} x\right)=y^{\prime} t^{\prime} x=s^{\prime}
$$

so that $s^{\prime} \in V(s)$. In particular, $\left(s, s^{\prime}\right)$ is an inverse pair in $S$. Finally $x s y^{\prime}=$ $x\left(x^{\prime} t y\right) y^{\prime}=\left(t t^{\prime}\right) t\left(t^{\prime} t\right)=t$.

Examples. (1) Let $T$ be a regular semigroup. It is easy to see that local submonoids of $T$ are always quasi-ideals. Let $e \in E(T)$. We claim that $T$ is an enlargement of $e T e$ if, and only if, $T=T e T$. By definition, $T$ is an enlargement of $e T e$ if, and only if, $T=T(e T e) T$. Thus if $T$ is an enlargement of $e T e$ then $T=T e T$. Conversely, if $T=T e T$ then $T=T e(T e T)=T(e T e) T$. In general, $T$ is an enlargement of a local submonoid if, and only if, $T=T u T$ for some $u \in T$. For if $T=T u T$, then $T=T\left(u u^{\prime} u\right) T$ where $u^{\prime} \in V(u)$. But $T u T \subseteq T u u^{\prime} T \subseteq T$. Hence $T=T\left(u u^{\prime}\right) T$. The converse is immediate.

Examples of regular semigroups $T$ for which there is an idempotent $e$ such that $T=T e T$ have been studied by a number of authors:

(i) If $T$ is a (0-)bisimple regular semigroup and $e$ is any nonzero idempotent then $T$ is an enlargement of $e T e$, which is a (0-)bisimple regular monoid [23], [41], [42].

(ii) An idempotent $e$ in a regular semigroup $T$ is said to be a mid-identity if $x y=x e y$ for all elements $x$ and $y$ in $T$. Clearly, $T=T e T$. Thus $T$ is an enlargement of $e$ Te. See [2] and [11].

(iii) An idempotent $e$ in a regular semigroup $T$ is said to be medial if for all $x \in I G(T)$ we have that $x=x e x$, where $I G(T)$ is the subsemigroup generated by the idempotents of $T$. Thus $I G(T)$ is an enlargement of $e I G(T) e$. It is also the case that $T$ is an enlargement of eTe since for all $x \in T$ and $x^{\prime} \in V(x)$, we have that $x=x\left(x^{\prime} x\right)=x\left(x^{\prime} x\right) e\left(x^{\prime} x\right)$. See [3], [4] and [32].

(iv) McAlister's Local Structure Theorem of [26] applies to regular semigroups having an idempotent $e$ such that $T=T e T$.

(v) For any regular semigroup $S$ the semigroup $\mathscr{Q}(S)$ of quasi-ideals is an enlargement of a meet semilattice with an identity [38].

(vi) Hotzel [12] considers semigroups $T$ such that $T=T e T$ within the context of actions of semigroups.

(vii) Talwar [44] proves, within the context of his Morita theory of a class of semigroups, that if $T=T e T$ then $T$ is Morita equivalent to $e T e$.

(2) Let $T$ be a regular semigroup and let $F$ be a set of idempotents of $T$ containing one idempotent from each $\mathscr{D}$-class of $T$. Put $S=\cup\{e T f: e, f \in F\}$. Then $T$ is an enlargement of $S$; it is clear that $S$ is a subsemigroup of $T$, it is regular by Result 1.6(iii), and it is easy to check that (E1), (E2) and (E3) hold. Compare this example with [1]. 
(3) Let $(Y, G, X)$ be a McAlister triple where both $X$ and $Y$ are semilattices [25]. Then $P(X, G, X)$ is an enlargement of $P(Y, G, X)$. See [18] for a discussion of this result.

(4) For every inverse semigroup $S$ there exists a factorisable inverse semigroup $F$ which contains $S$ as an inverse subsemigroup and such that $P \backslash U(F)$ is an enlargement of $S$. This result is proved in [17]. Example (3) above is an important ingredient in the proof.

(5) Let $T$ be a regular semigroup containing a regular subsemigroup $S$ which is a quasi-ideal such that $V(t) \cap S$ is non-empty for all $t$ in $T$. Then $T$ is an enlargement of $S$. This situation is studied in detail in [31]. See also [20].

We now consider the case where $S$ is a regular quasi-ideal of a regular semigroup $T$ but (E3) does not necessarily hold (examples of this situation may be found in [30]). Define

$$
\mathscr{E}_{T}(S)=\{t \in T: t \mathscr{D} s ; \text { some } s \in S\} .
$$

We have the following result.

Proposition 2.3. Let $S$ be a regular quasi-ideal of a regular semigroup T. Put $T^{\prime}=\mathscr{E}_{T}(S)$. Then

(i) $T^{\prime}$ is an order ideal of $T$.

(ii) $T^{\prime}=T S T$.

(iii) $T^{\prime}$ is a regular subsemigroup of $T$.

(iv) $T^{\prime}$ is an enlargement of $S$.

Proof. (i) Let $u \in T^{\prime}$, so that there exists $s \in S$ such that $u \mathscr{D} s$. Let $v \leq u$. Then by Result 1.7(i), there exists $s^{\prime} \leq s$ such that $v \mathscr{D} s^{\prime}$. But $S$ is an order ideal of $T$, so that $s^{\prime} \in S$. Thus $v \in T^{\prime}$.

(ii) Let $t \in T^{\prime}$. Then $t \mathscr{D} s$ some $s \in S$. Thus $t \mathscr{g} s$ and so $t=a s b$ some $a, b \in T$. Hence $T^{\prime} \subseteq T S T$. Conversely, if $t \in T S T$ then $t=a s b$ some $a, b \in T$ and $s \in S$. By Result 1.6(iv), we have that $a s b=a^{\prime} \cdot s^{\prime} \cdot b^{\prime}$ some $a^{\prime} \leq a, s^{\prime} \leq s$ and $b^{\prime} \leq b$. But then $t \mathscr{D} s^{\prime}$ and $s^{\prime} \in S$ since $S$ is an order ideal of $T$. Hence $t \in T^{\prime}$.

(iii) It is clear from the definition that $T^{\prime}$ is closed under trace products. By (i), $T^{\prime}$ is an order ideal of $T$, thus $T^{\prime}$ is a subsemigroup of $T$ by Result 1.6(iv). $T^{\prime}$ is a regular subsemigroup since if $u \in T^{\prime}$, then $V(u) \subseteq T^{\prime}$.

(iv) Clearly $S \subseteq T^{\prime}$. Thus $S$ is a quasi-ideal of $T^{\prime}$. (E3) holds from the definition of $T^{\prime}$. Thus $T^{\prime}$ is an enlargement of $S$.

The next result was suggested by a situation considered by McAlister [30].

Proposition 2.4. Let $T$ be an enlargement of $V$ and contain $U$ as a regular quasi-ideal. Put $V^{\prime}=\mathscr{E}_{T}(U) \cap V$. Then $\mathscr{E}_{T}(U)$ is an enlargement of $V^{\prime}$ and $U$. 
Proof. By Proposition 2.3, $\mathscr{E}_{T}(U)$ is an enlargement of $U$. Clearly, $V^{\prime} \subseteq \mathscr{E}_{T}(U) . V^{\prime}$ is non-empty, for if $u \in U$ then, since $T$ is an enlargement of $V$, there exists $v \in V$ such that $u \mathscr{D} v$ by (E3). In particular, $v \in V^{\prime}$. It remains to show that $\mathscr{E}_{T}(U)$ is an enlargement of $V^{\prime} . V^{\prime}$ is clearly a subsemigroup of $\mathscr{E}_{T}(U)$, and it is a regular subsemigroup since $\mathscr{E}_{T}(U)$ contains $V(x)$ if it contains $x$. (E1) holds because both $V$ and $\mathscr{E}_{T}(U)$ are order ideals of $T$. (E2) holds: let $x \in \mathscr{E}_{T}(U)$ such that for some $x^{\prime} \in V(x), x^{\prime} x, x x^{\prime} \in V^{\prime}$. Then in particular, $x^{\prime} x, x x^{\prime} \in V$ and so, since $T$ is an enlargement of $V$, it follows that $x \in V$. Thus $x \in V^{\prime}$ and (E2) holds. It is immediate that (E3) holds.

The significance of the following result will be discussed after Theorem 3.3.

Theorem 2.5. Let $T$ be an enlargement of a regular subsemigroup $S$.

(i) For any $Q \in \mathscr{Q}(S)$ we have that $Q T Q \in \mathscr{Q}(T)$.

(ii) For any $Q \in \mathscr{Q}(S)$ we have that $Q T Q=Q$.

(iii) If $Q \in \mathscr{Q}(T)$ and $Q \subseteq S$ then $Q \in \mathscr{Q}(S)$.

(iv) $\mathscr{Q}(T)$ is an enlargement of $\mathscr{Q}(S)$.

Proof. (i) Observe that $Q T$ and $T Q$ are respectively right and left ideals of $T$. Thus by Corollary 2.7 of [43], $Q T Q=Q T \cap T Q$ is a quasi-ideal of $T$.

(ii) $Q=Q S Q \subseteq Q T Q$, and

$$
Q T Q=Q(T S T) Q=(Q S Q)(T S T)(Q S Q)=Q(S(Q T S T Q) S) Q
$$

But $S(Q T S T Q) S \subseteq S T S=S$. Thus $Q T Q \subseteq Q S Q=Q$. Hence $Q=Q T Q$.

(iii) Let $Q$ be a quasi-ideal of $T$ contained in $S$. We claim that $Q$ is a quasi-ideal of $S$. Now $Q S Q \subseteq Q T Q=Q$. Since $Q=Q T Q$, if $q \in Q$ then we can write $q=q^{\prime} t q^{\prime \prime}$ where $q^{\prime}, q^{\prime \prime} \in Q \subseteq S$ and $t \in T$. But $q=q^{\prime}(e t f) q^{\prime \prime}$ where $e$ and $f$ are idempotents such that $e \mathscr{L} q^{\prime}$ in $S$ and $f \mathscr{R} q^{\prime \prime}$ in $S$. Hence etf $\in S$ and so $Q T Q \subseteq Q S Q$. It follows that $Q$ is a quasi-ideal of $S$.

(iv) From (i) and (ii) and Result 1.1 , we have that $\mathscr{Q}(S)$ is a regular subsemigroup of $\mathscr{2}(T)$. To show that $\mathscr{2}(T)$ is an enlargement of $2(S)$ we show that (E1), (E2) and (E3) hold. (E1) holds: let $Q \leq R \in \mathscr{Q}(S)$ in the Nambooripad order. Then by [38], we have that $Q \subseteq R \subseteq S$. Thus by (iii) $Q$ is a quasi-ideal of $S$. (E2) holds: suppose that $Q \in \mathcal{Q}(T)$ is such that there is $Q^{\prime} \in V(T)$ with $Q^{\prime} Q, Q Q^{\prime} \in \mathscr{Q}(S)$. Then

$$
Q=\left(Q Q^{\prime}\right) Q\left(Q^{\prime} Q\right) \subseteq S Q S \subseteq S T S \subseteq S
$$

Thus $Q$ is a quasi-ideal of $T$ contained in $S$ and so by (iii), $Q$ is a quasi-ideal of $S$. To show that (E3) holds we use Theorem 2.1(ii): let $Q \in \mathscr{Q}(T)$. Then $Q=Q T Q$. But $T=T S T$ thus $Q=(Q T) S(T Q)$. But $Q T, T Q \in \mathscr{Q}(T)$ and so $\mathscr{2}(T) \subseteq \mathscr{2}(T) \mathscr{L}(S) \mathscr{L}(T)$.

We now turn to the behaviour of enlargements under homomorphisms. 
Proposition 2.6. (i) Let $T$ be an enlargement of $S$ and let $\theta: T \rightarrow W$ be a surjective homomorphism. Then $W$ is an enlargement of $\theta(S)$.

(ii) Let $W$ be an enlargement of $T$ and $T$ be an enlargement of $S$. Then $W$ is an enlargement of $S$.

Proof. (i) Clearly, $\theta(S)$ is a regular subsemigroup of $W$. We show first that $\theta(S)$ is a quasi-ideal of $W$. Let $\theta(u), \theta(v) \in \theta(S)$ where $u, v \in S$, and let $w \in W$. Since $\theta$ is onto there is $t \in T$ such that $\theta(t)=w$. Thus $\theta(u) w \theta(v)=\theta(u t v)$. But $u t v \in S T S=S$ so that $\theta(u) w \theta(v) \in \theta(S)$. Hence $\theta(S) W \theta(S) \subseteq \theta(S)$, and so $\theta(S)$ is a quasi-ideal of $W$. We next show that $W \theta(S) W=W$. It is clearly enough to show that $W \subseteq W \theta(S) W$. Let $w \in W$ and $t \in T$ such that $\theta(t)=w$. Since $T=T S T$ we can write $t=t^{\prime} s t^{\prime \prime}$ where $t^{\prime}, t^{\prime \prime} \in T$ and $s \in S$. Thus

$$
w=\theta(t)=\theta\left(t^{\prime}\right) \theta(s) \theta\left(t^{\prime \prime}\right) \in W \theta(S) W .
$$

Thus $W$ is an enlargement of $\theta(S)$.

(ii) Straightforward.

Let $T$ be an enlargement of $S$. If $S$ has a zero, $z$, there is no guarantee that $z$ will be a zero of $T$. When it is, we shall say that $T$ is a 0 -enlargement of $S$. In the next result, we show how an enlargement of a semigroup $S$ with zero can be modified to produce a 0-enlargement of $S$.

Proposition 2.7. Let $S$ be a regular semigroup with zero $z$ and let $T$ be an enlargement of $S$. Put $Z=\{t \in T: t \mathscr{D} z\}$. Then $Z$ is an ideal of $T$, and the natural embedding $\imath: S \rightarrow T / Z$ is such that $T / Z$ is a 0-enlargement of $\imath(S)$.

Proof. $Z$ is an order ideal of $T$; for if $t \mathscr{D} z$ and $t^{\prime} \leq t$ then there exists $z^{\prime} \leq z$ such that $t^{\prime} \mathscr{D} z^{\prime}$ by Result 1.7(i). But $S$ is an order ideal of $T$ and so $z^{\prime} \in S$. Clearly $z$ is the smallest element in $S$ so that $z^{\prime}=z$. Hence $t^{\prime} \in Z$. To show that $Z$ is an ideal of $T$ containing $z$, let $x \in T$ and $t \in Z$. We can write $x t=x^{\prime} \cdot t^{\prime}$ where $x^{\prime} \leq x$ and $t^{\prime} \leq t$ by Result 1.6(iv). Since $Z$ is an order ideal we have that $t^{\prime} \in Z$, and $x t=x^{\prime} \cdot t^{\prime}$ implies that $x t \mathscr{D} t^{\prime}$. Thus $x t \mathscr{D} z$. Similarly, we have that $t x \in Z$. It is clear that $Z \cap S=\{z\}$. Thus the Rees quotient $T / Z$ contains a copy of $S$, which we identify with $S$. The zero of $S$ is now $Z$. It follows by Proposition 2.6(i), that $T / Z$ is an enlargement of $S$ and that the zero of $S$ is also the zero of $T$.

\section{Green's relations and ideal structure}

We begin with an elementary result which relates Green's relations on $S$ to those on an enlargement of $S$.

Lemma 3.1. If $T$ is an enlargement of $S$ then

(i) $\mathscr{L}(T) \cap(S \times S)=\mathscr{L}(S)$.

(ii) $\mathscr{R}(T) \cap(S \times S)=\mathscr{R}(S)$. 
(iii) $\mathscr{H}(T) \cap(S \times S)=\mathscr{H}(S)$.

(iv) If $(a, b) \in \mathscr{H}(T)$ and $a \in S$ then $b \in S$.

(v) $\mathscr{D}(T) \cap(S \times S)=\mathscr{D}(S)$.

(vi) $\mathscr{J}(T) \cap(S \times S)=\mathscr{J}(S)$.

Proof. (i) It is clearly enough to show that $\mathscr{L}(T) \cap(S \times S) \subseteq \mathscr{L}(S)$. Let $(a, b) \in$ $\mathscr{L}(T) \cap(S \times S)$. Then there exist $u, v \in T$ such that $u a=b$ and $v b=a$. Let $b^{\prime} \in V(b) \cap S$, so that $b^{\prime} b, b b^{\prime} \in S$. Similarly let $a^{\prime} \in V(a) \cap S$, so that $a^{\prime} a, a a^{\prime} \in S$. Then

$$
\left(\left(b b^{\prime}\right) u\left(a a^{\prime}\right)\right) a=b \quad \text { and } \quad\left(\left(a a^{\prime}\right) v\left(b b^{\prime}\right)\right) b=a .
$$

But $\left(b b^{\prime}\right) u\left(a a^{\prime}\right),\left(a a^{\prime}\right) v\left(b b^{\prime}\right) \in S$, so that $(a, b) \in \mathscr{L}(S)$.

(ii) Similar to the proof of (i) above.

(iii) By (i) and (iii).

(iv) Let $(a, b) \in \mathscr{H}(T)$ and $a \in S$. Then $a \mathscr{L}(T) B$ and $a \mathscr{R}(T) b$. As above we have elements $u, v, z$ and $y$ in $T$ such that

$$
u a=b \quad \text { and } \quad v b=a \text {, and } a z=b \text { and } b y=a .
$$

Let $a^{\prime} \in V(a) \cap S$. Then $(u a) \cdot a^{\prime} a=u a$ so that $b a^{\prime} a=b$. Similarly, $a a^{\prime} \cdot(a x)=a x$ so that $a a^{\prime} b=b$. It follows that $b \in S$.

(v) It is clearly enough to show that $\mathscr{D}(T) \cap(S \times S) \subseteq \mathscr{D}(S)$. Let $(a, b) \in \mathscr{D}(T)$ where $a, b \in S$. Let $a^{\prime} \in V(a) \cap S$ and $b^{\prime} \in V(b) \cap S$. Then $\left(a a^{\prime}, b b^{\prime}\right) \in \mathscr{D}(T)$. By Proposition II.3.6 [13], there exists $x \in T$ and $x^{\prime} \in V(x)$ such that $x x^{\prime}=a a^{\prime}$ and $x^{\prime} x=b b^{\prime}$. But then $a a^{\prime} \cdot x \cdot b b^{\prime}=x$. Thus $x \in S$. It follows that $\left(a a^{\prime}, b b^{\prime}\right) \in \mathscr{D}(S)$ and so $(a, b) \in \mathscr{D}(S)$.

(vi) It is clearly enough to show that $\mathscr{J}(T) \cap(S \times S) \subseteq \mathscr{J}(S)$. Thus suppose that $a, b \in S$ and $T a T=T b T$. We shall show that $S a S=S b S$. There exists elements $u, v, x, y \in T$ such that $a=u b v$ and $b=x a y$. Let $a^{\prime} \in V(a) \cap S$ and $b^{\prime} \in V(b) \cap S$. Then

$$
a=a a^{\prime} \cdot a \cdot a^{\prime} a=\left(a a^{\prime} u b b^{\prime}\right) b\left(b^{\prime} b v a^{\prime} a\right),
$$

and, similarly,

$$
b=\left(b b^{\prime} x a a^{\prime}\right) a\left(a^{\prime} a y b^{\prime} b\right)
$$

But

$$
a a^{\prime} u b b^{\prime}, b^{\prime} b v a^{\prime} a, b b^{\prime} x a a^{\prime}, a^{\prime} a y b^{\prime} b \in A,
$$

from which it follows that $S a S=S b S$.

The following result is the first comparing properties of $S$ with those of an enlargement.

Theorem 3.2. Let $T$ be an enlargement of $S$. Then $\mathscr{J}(S)=\mathscr{D}(S)$ if, and only if, $\mathscr{J}(T)=\mathscr{D}(T)$. 
Proof. Suppose that $\mathscr{J}(T)=\mathscr{D}(T)$. Then by Lemma 3.1(v) and (vi) we have that

$$
\mathscr{J}(S)=\mathscr{J}(T) \cap(S \times S)=\mathscr{D}(T) \cap(S \times S)=\mathscr{D}(S) .
$$

Thus $\mathscr{J}(S)=\mathscr{D}(S)$ as required. Conversely, suppose that $\mathscr{J}(S)=\mathscr{D}(S)$. We show that $\mathscr{J}(T) \subseteq \mathscr{D}(T)$. Suppose that $(u, v) \in \mathscr{J}(T)$, and let $u \mathscr{D}(T) a$ and $v \mathscr{D}(T) b$ where $a, b \in S$. Then $(a, b) \in \mathscr{J}(T) \cap(S \times S)=\mathscr{J}(S)$ by Lemma 3.1(vi). But by assumption $(a, b) \in \mathscr{D}(S)$. Hence $(u, v) \in \mathscr{D}(T)$.

The following result was motivated by analogous results in the Morita theory of rings.

Theorem 3.3. Let $T$ be an enlargement of a regular subsemigroup $S$. Then

(i) The lattice of two-sided ideals of $T$ is order isomorphic to the lat tice of two-sided ideals of $S$.

(ii) The posets $T / \mathscr{J}$ and $S / \mathscr{J}$ are order isomorphic.

Proof. (i) Let $J$ be an ideal of $S$. Then clearly $T J T$ is an ideal of $T$. We claim that $T J T \cap S=J$. It is clear that $J \subseteq T J T \cap S$. Let $x \in T J T \cap S$. Then $x=u k v$ where $u, v \in T$ and $k \in J$. Since $x \in S$, a regular subsemigroup, it is possible to find $e, f \in E(S)$ such that $x=e x f$. Similarly, it is possible to find $e^{\prime}, f^{\prime} \in E(S)$ such that $k=e^{\prime} k f^{\prime}$. Thus $x=\left(e u e^{\prime}\right) k\left(f^{\prime} v f\right)$. But $e u e^{\prime}, f^{\prime} v f \in S$ and so it follows that $x \in J$, as required.

Denote the lattices of ideals of $S$ and $T$ by $\mathscr{I}(S)$ and $\mathscr{I}(T)$ respectively. Define functions $\alpha: \mathscr{I}(S) \rightarrow \mathscr{I}(T)$ and $\beta: \mathscr{I}(T) \rightarrow \mathscr{I}(S)$ by $\alpha(J)=T J T$ and $\beta(M)=M \cap S$. It is clear that these functions are well-defined. It is also clear that both are isotone. We showed above that the composition $\beta \alpha$ is the identity of $\mathscr{I}(S)$. We now show that $\alpha \beta$ is the identity on $\mathscr{F}(T)$. Let $M$ be an ideal of $T$. It is clear that $T(M \cap S) T \subseteq M$. We show that the reverse containment holds. Let $m \in M$ and $m^{\prime} \in V(m)$. By Lemma 2.2, we can find inverse pairs $\left(x, x^{\prime}\right)$ and $\left(y, y^{\prime}\right)$ in $T$ and an inverse pair $\left(s, s^{\prime}\right)$ in $S$ such that

$$
m=x \cdot s \cdot y^{\prime}, x x^{\prime}=m m^{\prime}, y y^{\prime}=m^{\prime} m, x^{\prime} x=s s^{\prime} \quad \text { and } \quad y^{\prime} y=s^{\prime} s .
$$

It follows that $s=x^{\prime} \cdot m \cdot y \in S$. But $m \in M$ is an ideal of $T$ so that $s \in M$. Hence $m \in T(M \cap S) T$. It follows that $\alpha \beta$ is the identity on $\mathscr{I}(T)$. Thus $\mathscr{I}(S)$ and $\mathscr{I}(T)$ are order isomorphic.

(ii) We show that the functions $\alpha$ and $\beta$ defined in (i) above map principal ideals to principal ideals. Let $a \in S$. By definition, $\alpha(S a S)=T(S a S) T$. We claim that $\alpha(S a S)=T a T$. Clearly, $T(S a S) T \subseteq T a T$. On the other hand, let $e, f \in E(S)$ such that $a=e a f$. Then

$$
T a T=(T S T)(e a f)(T S T)=T((S T e) a(f T S)) T \subseteq T(S a S) T
$$

Thus $\alpha(\operatorname{SaS})=T a T$. Now consider $\beta(T a T)=\operatorname{TaT} \cap S$, where $a \in T$. We claim that $\beta(T a T)=S b S$ where $a \mathscr{D}(T) b \in S$. Clearly, since $a \mathscr{D}(T) b$, we have that $T a T=T b T$. We 
show that $T b T \cap S=S b S$. Clearly, $S b S \subseteq T b T \cap S$. So let $s \in T b T \cap S$. Then $s=u b v$ where $u, v \in T$. But $s, b \in S$, so there are $e, f, i, j \in E(S)$ such that $s=e s f$ and $b=i b j$. Thus $s=(e u i) b(j v f)$, where eui,jof $\in S$, and so $s \in S b S$.

Remark. There is an alternative way of proving (i) above. If $T$ is an enlargement of $S$ then $\mathscr{Q}(T)$ is an enlargement of $\mathscr{Q}(S)$. Since $S \in V(T)$ in $\mathscr{Q}(T)$ we have that $T \mathscr{Q}(T) T$ is isomorphic to $S 2(S) S$ by Result 1.4 . But by [38], $T 2(T) T$ is the lattice of all twosided ideals of $T$ and $S \mathscr{Q}(S) S$ is the lattice of all two-sided ideals of $S$.

Certain classes of regular semigroups are closed under the formation of enlargements or 0-enlargements.

Theorem 3.4. Let $T$ be an enlargement of a regular subsemigroup $S$. Then

(i) $S$ is combinatorial if, and only if, $T$ is combinatorial.

(ii) $S$ is bisimple if, and only if, $T$ is bisimple.

(iii) $S$ is simple if, and only if, $T$ is simple.

If $T$ is a 0 -enlargement of $S$. Then

(iv) $S$ is 0-bisimple if, and only if, $T$ is 0-bisimple.

(v) $S$ is 0 -simple if, and only if, $T$ is 0 -simple.

Proof. (i) Suppose that $S$ is combinatorial. Let $(x, y) \in \mathscr{H}(T)$. Since $T$ is regular there are idempotents $e$ and $f$ such that $e \mathscr{R} \times \mathscr{L} f$ and $e \mathscr{R} y \mathscr{L} f$. By Lemma 2.2, let $x^{\prime} \in V(x)$ and $y^{\prime} \in V(y)$ be such that $x x^{\prime}=e=y y^{\prime}$ and $x^{\prime} x=f=y^{\prime} y$, and let $u, v \in T$ and $u^{\prime} \in V(u)$ and $v^{\prime} \in V(v)$ be such that $u u^{\prime}=e$ and $v v^{\prime}=f$ and $u^{\prime} u, v^{\prime} v \in E(S)$. It is clear that $u^{\prime} y v \mathscr{H}(T) u^{\prime} x v$. Now $u^{\prime} y v, u^{\prime} x v \in S$, since $u^{\prime} u, v^{\prime} v \in E(S)$. Thus $u^{\prime} y v \mathscr{H}(S) u^{\prime} x v$ by Lemma 3.1(iii). But $S$ is combinatorial so that $u^{\prime} y v=u^{\prime} x v$. Hence $x=y$. It follows that $T$ is combinatorial. Conversely, it is immediate by Lemma 3.1 (iii) that if $T$ is combinatorial then $S$ is combinatorial.

(ii) Suppose that $S$ is bisimple. Since $T$ is an enlargement of $S$ every element of $T$ is $\mathscr{D}$-related to an element of $S$. Hence the result. Conversely, suppose that $T$ is bisimple. Let $e, f \in E(S)$. Then since $T$ is bisimple there is a $t \in T$ and $t^{\prime} \in V(t)$ such that $t^{\prime} t=e$ and $t t^{\prime}=f$. But then $f t e=t$, so that $t \in S$. Thus $S$ is bisimple.

(iii) This follows from Theorem 3.3(i).

(iv) Let $T$ be a 0 -enlargement of $S$. Suppose that $S$ is 0 -bisimple. Then every element of $T$ is $\mathscr{D}$-related to 0 or an element of $S \backslash\{0\}$. Since 0 is also the zero of $T$, it follows that $T$ is 0 -bisimple. Conversely, if $T$ is 0 -bisimple then $S$ is 0 -bisimple by a similar argument to (ii) above.

(v) Let $T$ be a 0 -enlargement of $S$. Suppose that $S$ is 0 -simple. Then by Theorem 3.3 , the only ideals of $T$ are $\{0\}$ and $T$ itself. Clearly, since $S^{2}$ is not $\{0\}$, nor is $T^{2}$. Thus $T$ is 0 -simple. Conversely, suppose that $T$ is 0 -simple. Then the only ideals of $S$ are $\{0\}$ and $S$ itself. Since $S$ is regular and $S \neq\{0\}$ we have immediately that $S^{2} \neq\{0\}$. 


\section{Local structure}

We begin with a simple result which shows that if $T$ is an enlargement of $S$ then every local submonoid of $T$ is isomorphic to one of $S$.

Lemma 4.1. (i) Let $T$ contain a regular quasi-ideal $S$. If $e \in E(T)$ is such that $e \mathscr{D} f$, where $f \in E(S)$, then $e T e$ is isomorphic to $f S f$.

(ii) If $T$ is an enlargement of $S$ then every local submonoid of $T$ is isomorphic to a local submonoid of $S$.

Proof. (i) By Result 1.4, eTe is isomorphic to $f T f$. But $f T f \subseteq S T S \subseteq S$. Thus $f T f \subseteq f S f$ and so $f T f=f S f$. The proof of (ii) is now immediate.

In [26, Theorem 2.4], McAlister proved his Local Structure Theorem and showed that for every regular semigroup $T$ and $e \in E(T)$ such that $T=T e T$ then $T$ is a locally isomorphic image of a regular Rees matrix semigroup over $e T e$. We generalise this result below.

Theorem 4.2. Let $T$ be an enlargement of $U$ and $V$. Then $V$ is a locally isomorphic image of a regular Rees matrix semigroup over $U$.

Proof. We begin by defining the ingredients of $M=M(U ; I, \Lambda ; p) . T$ is an enlargement of $U$ so that for each $e \in E(V)$ we can choose elements $r_{e}$ and $r_{e}^{\prime} \in V\left(r_{e}\right)$ such that $r_{e} r_{e}^{\prime}=e$ and $r_{e}^{\prime} r_{e} \in E(U)$ by (E3). If $e \in E(U)$ then choose $r_{e}=r_{e}^{\prime}=e$. Put $I=\Lambda=E(V)$ and define $p_{i j}=r_{i}^{\prime} r_{j}$ : this is well-defined, for $\left(r_{i}^{\prime} r_{i}\right) p_{i j}\left(r_{j}^{\prime} r_{j}\right)=p_{i j}$ and $r_{i}^{\prime} r_{i}, r_{j}^{\prime} r_{j} \in E(U)$ and so $p_{i j} \in U$, since $U$ is a quasi-ideal of $T$.

Now define a function $\theta: R M(U ; I, \Lambda ; p) \rightarrow V$ by $\theta(i, x, j)=r_{i} x r_{j}^{\prime}$. This is well-defined since $V$ is a quasi-ideal of $T$ and $\left(r_{i} r_{i}^{\prime}\right)\left(r_{i} x r_{j}^{\prime}\right)\left(r_{j} r_{j}^{\prime}\right)=r_{i} x r_{j}^{\prime}$ and $r_{i} r_{i}^{\prime}, r_{j} r_{j}^{\prime} \in V$. Thus $r_{i} x r_{j}^{\prime} \in V$. It is easy to check that $\theta$ is a homomorphism. Next we show that $\theta$ is onto. Let $v \in V$ and choose $v^{\prime} \in V(v) \cap V$. Put $v v^{\prime}=i$ and $v^{\prime} v=j$ and put $u=r_{i}^{\prime} v r_{j}$. Then $u \in U$ and $v=r_{i}\left(r_{i}^{\prime} v r_{j}\right) r_{j}^{\prime}$. If we show that $\left(i, r_{i}^{\prime} v r_{j}, j\right)$ is regular then $\theta\left(i, r_{i}^{\prime} v r_{j}, j\right)=v$. By Result 1.6(iii), the set $V\left(r_{i}^{\prime} v r_{j}\right) \cap r_{j}^{\prime} T r_{i}$ is non-empty. Let $r_{j}^{\prime} z r_{i} \in V\left(r_{i}^{\prime} v r_{j}\right)$. Then $r_{j}^{\prime} z r_{i} \in U$, so that $\left(j, r_{j}^{\prime} z r_{i}, i\right) \in M$ and

$$
\left(i, r_{i}^{\prime} v r_{j}, j\right)\left(j, r_{j}^{\prime} z r_{i}, i\right)\left(i, r_{i}^{\prime} v r_{j}, j\right)=\left(i, r_{i}^{\prime} v r_{j}, j\right)
$$

Thus $\left(i, r_{i}^{\prime} v r_{j}, j\right) \in R M$ as required. Finally, we show that $\theta$ is a local isomorphism. Let $(i, x, j)$ be an idempotent, so that $x=x p_{j i} x$. Let

$$
(i, u, j),(i, v, j) \in(i, x, j) R M(i, x, j) \text { with } \theta(i, u, j)=\theta(i, v, j)
$$

Then $r_{i} u r_{j}^{\prime}=r_{i} v r_{j}^{\prime}$ and

$$
u p_{j i} x=x p_{j i} u=u \text { and } v p_{j i} x=x p_{j i} v=v
$$


But then

$$
u=\left(x p_{j i}\right) u\left(p_{j i} x\right)=x r_{j}^{\prime}\left(r_{i} u r_{j}^{\prime}\right) r_{i} x=x r_{j}^{\prime}\left(r_{i} v r_{j}^{\prime}\right) r_{i} x=\left(x p_{j i}\right) v\left(p_{j i} x\right)=v
$$

The proofs of the following corollaries are now immediate.

Corollary 4.3. Let $T$ be an enlargement of $S$. Then $T$ is a locally isomorphic image of a regular Rees matrix semigroup over $S$.

McAlister's Local Structure Theorem [26] is the following.

Corollary 4.4. Let $S$ be a regular semigroup having an idempotent $e$ such that $S=S e S$. Then $S$ is a locally isomorphic image of a regular Rees matrix semigroup over eSe.

\section{Congruences}

We begin by defining a new equivalence relation on the lattice of all congruences $\mathscr{C}(S)$ of a regular semigroup $S$.

Definition. For $\alpha, \beta \in \mathscr{C}(S)$, we say that $\alpha$ is locally equal to $\beta$, denoted by $\alpha \sim \beta$, if $\alpha \cap(e S f \times e S f)=\beta \cap(e S f \times e S f)$ for all $e, f \in E(S)$.

Proposition 5.1. (i) The relation $\sim$ defined above is a lattice congruence on $\mathscr{C}(S)$.

(ii) The congruences $\sim$-related to the identity congruence $\Delta$ on $S$ lie in the interval $[\Delta, \rho]$, where $\rho$ is the congruence of Result 1.12.

(iii) If $\alpha$ and $\beta$ are idempotent separating congruences on $S$ then $\alpha \sim \beta$ implies that $\alpha=\beta$.

Proof. (i) It is clear that $\sim$ is an equivalence relation. To prove that $\sim$ is a lattice congruence on $\mathscr{C}(S)$ we have to show that for all $\alpha, \beta, \gamma, \delta \in \mathscr{C}(S)$ we have that

$$
\alpha \sim \beta \text { and } \gamma \sim \delta \text { implies }(\alpha \wedge \gamma) \sim(\beta \wedge \delta) \text { and }(\alpha \vee \gamma) \sim(\beta \vee \delta) \text {. }
$$

The former result is straightforward since $\alpha \wedge \gamma=\alpha \cap \gamma$. The latter result is proved as follows. Let $(a, b) \in(\alpha \vee \gamma) \cap(e S f \times e S f)$ where $e$ and $f$ are idempotents. By Proposition I.5.14 of [13], there exists a sequence of elements $x_{1}, \ldots, x_{2 n-1}$ in $S$ such that

$$
\left(a, x_{1}\right) \in \alpha,\left(x_{1}, x_{2}\right) \in \gamma,\left(x_{2}, x_{3}\right) \in \alpha, \ldots,\left(x_{2 n-1}, b\right) \in \gamma .
$$

Since $\alpha$ and $\gamma$ are congruences, and since $e a f=a$ and $e b f=b$, we have that

$$
\left(a, e x_{1} f\right) \in \alpha,\left(e x_{1} f, e x_{2} f\right) \in \gamma,\left(e x_{2} f, e x_{3} f\right) \in \alpha, \ldots,\left(e x_{2 n-1} f, b\right) \in \gamma
$$


By assumption,

$$
\alpha \cap(e S f \times e S f)=\beta \cap(e S f \times e S f) \text { and } \gamma \cap(e S f \times e S f)=\delta \cap(e S f \times e S f) .
$$

Thus we have that

$$
\left(a, e x_{1} f\right) \in \beta,\left(e_{1} f, e x_{2} f\right) \in \delta,\left(e_{2} f, e x_{3} f\right) \in \beta, \ldots,\left(\operatorname{ex}_{2 n-1} f, b\right) \in \delta
$$

Hence $(a, b) \in(\beta \vee \delta) \cap(e S f \times e S f)$. By symmetry we obtain the result.

(ii) Let $\alpha \sim \Delta$. Then $\alpha \cap(e S f \times e S f)=\Delta \cap(e S f \times e S f)$ for all idempotents $e$ and $f$ in $S$. Thus $\alpha$ is a locally isomorphic congruence and so by Proposition 1.2 of [28] we have that $\alpha \subseteq \rho$.

(iii) Let $\alpha$ and $\beta$ be idempotent separating congruences on $S$ such that $\alpha \sim \beta$. Let $(a, b) \in \alpha$. Then, in particular, $(a, b) \in \mathscr{H}$, and so there are idempotents $e$ and $f$ in $S$ such that $e \mathscr{R} a \mathscr{L} f$ and $e \mathscr{R} b \mathscr{L} f$. Thus $a, b \in e S f$. Hence

$$
(a, b) \in \alpha \cap(e S f \times e S f)=\beta \cap(e S f \times e S f),
$$

and so $(a, b) \in \beta$. By symmetry we obtain $\alpha=\beta$.

The definition of locally equal congruences provides a neat way of expressing some properties of congruences in enlargements.

Proposition 5.2. Let $T$ be an enlargement of $S$.

(i) If $\alpha$ and $\beta$ are congruences on $T$ such that

$$
\alpha \cap(S \times S) \sim \beta \cap(S \times S)
$$

in $S$ then $\alpha \sim \beta$ in $T$.

(ii) Let $\alpha$ be an idempotent separating congruence on $S$. Suppose that $\beta$ and $\gamma$ are idempotent separating congruences on $T$ such that

$$
\beta \cap(S \times S)=\alpha=\gamma \cap(S \times S) .
$$

Then $\beta=\gamma$.

Proof. (i) Let $(x, y) \in \alpha \cap(e T f \times e T f)$. By Lemma 2.2, choose elements $a, b$ in $T$ and elements $a^{\prime} \in V(a)$ and $b^{\prime} \in V(b)$ such that $a a^{\prime}=e, b b^{\prime}=f$ and $a^{\prime} a, b^{\prime} b \in S$. Since $\alpha$ is a congruence we have that $\left(a^{\prime} x b, a^{\prime} y b\right) \in \alpha$. Clearly, $a^{\prime} x b, a^{\prime} y b \in S$, and so

$$
\left(a^{\prime} x b, a^{\prime} y b\right) \in \alpha \cap\left(a^{\prime} a S b^{\prime} b \times a^{\prime} a S b^{\prime} b\right) .
$$

Thus by assumption

$$
\left(a^{\prime} x b, a^{\prime} y b\right) \in \beta \cap\left(a^{\prime} a S b^{\prime} b \times a^{\prime} a S b^{\prime} b\right) .
$$


But then $(e x f, e y f) \in \beta$ so that $(x, y) \in \beta \cap(e T f \times e T f)$. Thus

$$
\alpha \cap(e T f \times e T f) \subseteq \beta \cap(e T f \times e T f) .
$$

The reverse inclusion follows by a symmetric argument.

(ii) Since $\beta \cap(S \times S)=\alpha=\gamma \cap(S \times S)$, we certainly have that

$$
\beta \cap(S \times S) \sim \gamma \cap(S \times S) .
$$

Thus by (i), $\beta \sim \gamma$. Hence by Proposition 5.1(iii) we have that $\beta=\gamma$.

We now consider the case of idempotent separating congruences in more detail.

Proposition 5.3. Let $T$ be an enlargement of $S$. Then

(i) Every idempotent separating congruence on $S$ extends uniquely to an idempotent separating congruence on $T$.

(ii) If $\alpha$ is an idempotent separating congruence on $S$ then its unique extension $\beta$ to $T$ is defined as follows: $(x, y) \in \beta$ if, and only if, for some $x^{\prime} \in V(x)$ and $y^{\prime} \in V(y)$ we have that $x x^{\prime}=y y^{\prime}$ and $x^{\prime} x=y^{\prime} y$ and there exist $a, b \in T, a^{\prime} \in V(a)$ and $b^{\prime} \in V(b)$ such that $a a^{\prime}=x x^{\prime}, b b^{\prime}=x^{\prime} x, a^{\prime} a, b^{\prime} b \in S$ and $\left(a^{\prime} x b, a^{\prime} y b\right) \in \alpha$.

Proof. (i) Since $S$ is a quasi-ideal of $T$, every idempotent separating congruence $\alpha$ on $S$ generates an idempotent separating congruence on $T$ extending $\alpha$ by Proposition 2.4 of [27]. Uniqueness follows from Proposition 5.2(ii).

(ii) Since $\beta$ is an idempotent separating congruence we have that

$$
(x, y) \in \beta \Leftrightarrow(x, y) \in \beta \text { and }(x, y) \in \mathscr{H} \text {. }
$$

But

$$
(x, y) \in \mathscr{H} \Leftrightarrow x x^{\prime}=y y^{\prime} \quad \text { and } x^{\prime} x=y^{\prime} y \text { some } x^{\prime} \in V(x) \text { and } y^{\prime} \in V(y)
$$

By Lemma 2.3, we can find $a, b \in T, a^{\prime} \in V(a), b^{\prime} \in V(b)$ such that

$$
a a^{\prime}=x x^{\prime}, b b^{\prime}=x^{\prime} x, a^{\prime} a, b^{\prime} b \in S .
$$

Clearly $\left(a^{\prime} x b, a^{\prime} y b\right) \in \beta$. But $a^{\prime} x b, a^{\prime} y b \in S$ and. so $\left(a^{\prime} x b, a^{\prime} y b\right) \in \alpha$. The converse is straightforward.

A corollary of the above result is Lemma 3 of [9].

We denote by $\mathscr{C}_{i s}(S)$ the lattice of idempotent separating congruences on a regular semigroup $S$.

Theorem 5.4. Let $T$ be an enlargement of a regular semigroup $S$. Then $\mathscr{C}_{i s}(S)$ and $\mathscr{C}_{\text {is }}(T)$ are isomorphic lattices. 
Proof. Define functions $\mathfrak{E}: \mathscr{C}(S) \rightarrow \mathscr{C}(T)$ and $\Re: \mathscr{C}(T) \rightarrow \mathscr{C}(S)$ as follows: $\mathscr{E}(\alpha)$ is the congruence defined in Proposition 5.3(i), and $\mathfrak{R}(\beta)=\beta \cap(S \times S)$. $\mathcal{E}$ is well-defined by Proposition 5.3(i), whilst it is immediate that $\Re$ is well-defined. $\mathfrak{E}$ and $\mathfrak{R}$ are mutually inverse for by Propostion 5.3(i), we have that $\Re(\mathscr{E}(\alpha))=\alpha$, and by Proposition 5.2(ii), $\mathfrak{E}(\mathfrak{R}(\beta))=\beta$ holds. It is immediate from the definitions that $\mathbb{E}$ and $\Re$ are isotone.

The following is now immediate. Note that it also follows from Corollary 6 of [8].

Corollary 5.5. Let $T$ be an enlargement of $S$. Then $S$ is fundamental iff $T$ is fundamental.

We conclude this section with an interpretation of the relation of local equality on the lattice $\mathscr{C}(S)$ of all congruences of the regular semigroup $S$. We may define a category.

$$
C(S)=\{(e, x, f): \operatorname{exf}=f \quad \text { where } \quad e, f \in E(S) \text { and } \quad x \in S\},
$$

with identities $\{(e, e, e): e \in E(S)\}$ and partial product defined by $(e, x, f)(i, y, j)=$ $(e, x y, j)$ if $f=i$ and undefined otherwise. Every semigroup congruence $\alpha$ on $S$ defines a category congruence (see MacLane [21]) $\alpha^{\prime}$ on $C(S)$ by $(e, x, f) \alpha^{\prime}(i, y, j) \Leftrightarrow e=i, f=j$ and $x \alpha y$. It is now clear that $\alpha \sim \beta$ if, and only if, $\alpha$ and $\beta$ induce the same category congruence on $C(S)$.

\section{Inverse enlargements of inverse semigroups}

In this section, we examine in more detail the case where both $S$ and its enlargement $T$ are inverse. We begin with some results on arbitrary congruences.

Proposition 6.1. Let $S$ be an inverse semigroup. Then for any two congruences $\alpha$ and $\beta$ on $S$, we have that $\alpha \sim \beta$ if, and only if, $\alpha=\beta$.

Proof. Let $\alpha \sim \beta$. Then $\alpha \cap(e S f \times e S f)=\beta \cap(e S f \times e S f)$ for all $e, f \in E(S)$. Suppose that $(x, y) \in \alpha$. Then

$$
\left(x, x x^{-1} y x^{-1} x\right) \in \alpha \cap\left(\left(x x^{-1} S x^{-1} x\right) \times\left(x x^{-1} S x^{-1} x\right)\right) .
$$

Thus, by assumption,

$$
\left(x, x x^{-1} y x^{-1} x\right) \in \beta \cap\left(\left(x x^{-1} S x^{-1} x\right) \times\left(x x^{-1} S x^{-1}\right)\right) .
$$

Hence $\beta(x) \leq \beta(y)$, where $\beta(x)$ is the $\beta$-congruence class containing $x$. A similar argument shows that $\beta(y) \leq \beta(x)$, and so $(x, y) \in \beta$. Thus $\alpha \subseteq \beta$. By symmetry, $\beta \subseteq \alpha$ and so $\alpha=\beta$. 
We do not know which other classes of regular semigroups have the property that $\sim$ is the equality relation.

Corollary 6.2. Let $T$ be an inverse enlargement of an inverse subsemigroup $S$. Suppose $\tau$ and $\rho$ are congruences on $T$ such that $\tau \cap(S \times S)=\rho \cap(S \times S)$. Then $\tau=\rho$.

Proof. Clearly $\tau \cap(S \times S) \sim \rho \cap(S \times S)$, and so by Proposition 5.2(i), $\tau \sim \rho$ on $T$. But $T$ is inverse and so the result follows by Proposition 6.1.

In the proof of the following, we use an idea due to Pedro da Silva in Chapter IV of [6].

Theorem 6.3. Let $T$ be an inverse enlargement of an inverse subsemigroup $S$. Let $\sigma$ be a congruence on $S$ and let $\rho$ be the congruence on $T$ generated by $\sigma$. Then $\rho \cap(S \times S)=\sigma$.

Proof. Let $(a, b) \in \rho$ where $a, b \in S$. Then there is a sequence of elementary $\sigma$ transitions.

$$
a=z_{1} \rightarrow z_{2} \rightarrow \ldots \rightarrow z_{n}=b,
$$

where $z_{i} \rightarrow z_{i+1}(1 \leq i \leq n-1)$ is an elementary $\sigma$-transition, such that

$$
z_{i}=x_{i} p_{i} y_{i}, z_{i+1}=x_{i} q_{i} y_{i} \text { and }\left(p_{i}, q_{i}\right) \in \sigma \text {. }
$$

We show that there are elements $a=w_{1}, \ldots, w_{n} \in S$ such that $w_{i} \leq z_{i}$ and $\sigma\left(w_{1}\right)=\ldots=\sigma\left(w_{n}\right)$. This implies that $\sigma(a) \leq \sigma(b)$ and symmetry yields $\sigma(a)=\sigma(b)$. Suppose that we have constructed elements $w_{1}, \ldots, w_{r}$ satisfying the above conditions. We show how to construct $w_{r+1}$. Consider the elementary $\sigma$-transition $z_{r} \rightarrow z_{r+1}$. Since $w_{r} \leq z_{r}$ we have that $w_{r}=\left(e x_{r} i\right) p_{r}\left(j y_{r} f\right)$ where $e, i, j, f \in E(S)$ and $e \mathscr{R} w_{r}, i \mathscr{R} p_{r}, j \mathscr{L} p_{r}$ and $f \mathscr{L} w_{r}$. It follows that ex, $i, j y_{r} f \in S$. Define $w_{r+1}=\left(e x_{r} i\right) q_{r}\left(j y_{r} f\right)$. Then $w_{r+1} \in S$, $\left(w_{r}, w_{r+1}\right) \in \sigma$, and $w_{r+1} \leq z_{r+1}$.

We now have the following result.

Theorem 6.4. Let $T$ be an inverse enlargement of an inverse subsemigroup $S$. Then

(i) The lattices of all congruences of $S$ and $T$ are isomorphic.

(ii) The isomorphism induces an isomorphism between the lattices of idempotent separating congruences.

(iii) The isomorphism induces an isomorphism between the lattices of group congruences.

Proof. (i) Let $\mathscr{C}(S)$ and $\mathscr{C}(T)$ be the lattices of congruences of $S$ and $T$ respectively. Define functions $\mathfrak{E}: \mathscr{C}(S) \rightarrow \mathscr{C}(T)$ and $\mathfrak{R}: \mathscr{C}(T) \rightarrow \mathscr{C}(S)$ where $\mathscr{E}(\rho)$ is the congruence 
on $T$ generated by $\rho$, and $\Re(\tau)=\tau \cap(S \times S)$. Clearly both $\mathcal{E}$ and $\Re$ are isotone. Theorem 6.3 shows precisely that $\mathfrak{R E}$ is the identity, whereas Corollary 6.2 shows that $\mathfrak{E} \Re$ is the identity. Thus the lattices $\mathscr{C}(S)$ and $\mathscr{C}(T)$ are isomorphic.

(ii) If $\rho$ is an idempotent separating congruence on $S$, then $\mathcal{E}(\rho)$ is an idempotent separating congruence on $T$ by Proposition 5.3. On the other hand, if $\tau$ is idempotent separating $T$ then $\mathfrak{R}(\tau)$ is clearly idempotent separating on $S$. It follows that $\mathfrak{E}$ and $\mathfrak{R}$ reduce to an isomorphism of the lattices of idempotent separating congruences.

(iii) Let $\sigma(T)$ and $\sigma(S)$ be the minimum group congruences on $T$ and $S$ respectively. It is enough to show that $\sigma(T) \cap(S \times S)=\sigma(S)$. But this is immediate from the fact that $S$ is an order ideal of $T$.

In the remainder of this section, we shall show how to construct all inverse enlargements of an inverse monoid $S$. We begin with some elementary properties of local isomorphisms onto inverse semigroups.

Proposition 6.5. Let $S$ be regular, $T$ inverse and $\theta: S \rightarrow T$ a surjective local isomorphism. Then

(i) For all $x \in S$ and $x^{\prime} \in V(x)$, we have that $\theta\left(x^{\prime}\right)=\theta(x)^{-1}$.

(ii) For all $x, y \in S, \theta(x y x)=\theta(x)$ implies that $x y x=x$.

(iii) For all $e \in E(T), \theta^{-1}(e) \subseteq E(S)$.

Proof. (i) If $x^{\prime} \in V(x)$, then $x x^{\prime} x=x$ and $x^{\prime} x x^{\prime}=x^{\prime}$, and so

$$
\theta(x) \theta\left(x^{\prime}\right) \theta(x)=\theta(x) \text { and } \theta\left(x^{\prime}\right) \theta(x) \theta\left(x^{\prime}\right)=\theta\left(x^{\prime}\right)
$$

But $T$ is inverse. Thus $\theta\left(x^{\prime}\right)=\theta(x)^{-1}$.

(ii) Let $x^{\prime} \in V(x)$. Then

$$
x x^{\prime}(x y x) x^{\prime} x=x y x \text { and } x x^{\prime} \cdot x \cdot x^{\prime} x=x
$$

so that $x y x, x \in x x^{\prime} S x^{\prime} x$. But $\theta(x y x)=\theta(x)$. Thus by result 1.9 , we have that $x y x=x$.

(iii) Let $\theta(x)=e$. Then $\theta\left(x^{2}\right)=\theta(x)^{2}=e^{2}=e$. Let $x^{\prime} \in V(x)$. Then

$$
\theta(x)=\theta\left(x^{2}\right)=\theta\left(x\left(x^{\prime} x\right) x\right)
$$

Thus by (ii) above, we have that $x^{2}=x$.

We now have the following result, important for our final construction.

Theorem 6.6. (i) Let $S$ be orthodox, $T$ inverse, and $\theta: S \rightarrow T$ a surjective local isomorphism. Then $k e r \theta=\gamma$.

(ii) Let $S$ be a generalised inverse semigroup. Then $S$ has a unique locally isomorphic inverse congruence, namely $\gamma$. 
Proof. (i) Since $\gamma$ is the minimum inverse semigroup congruence we have that $\gamma \subseteq \operatorname{ker} \theta$. We now show that $\operatorname{ker} \theta \subseteq \gamma$. Let $\theta(x)=\theta(y)$. By Result 1.8, we need to show that $V(x)=V(y)$. Let $x^{\prime} \in V(x)$. Then $\theta\left(y x^{\prime} y\right)=\theta(y) \theta\left(x^{\prime}\right) \theta(y)$. But by Proposition 6.5(i), $\theta\left(x^{\prime}\right)=\theta(x)^{-1}$. Thus

$$
\theta\left(y x^{\prime} y\right)=\theta(y) \theta(x)^{-1} \theta(y)=\theta(y) \theta(y)^{-1} \theta(y)
$$

since $\theta(x)=\theta(y)$. It follows that $\theta\left(y x^{\prime} y\right)=\theta(y)$, and so by Proposition 6.5(ii), we have that $y x^{\prime} y=y$. Similarly, $x^{\prime} y x^{\prime}=x^{\prime}$. Thus $x^{\prime} \in V(y)$ and so $V(x) \subseteq V(y)$. By symmetry, $V(y) \subseteq V(x)$. Hence $V(x)=V(y)$.

(ii) By Result 1.10, $\gamma$ is a locally isomorphic inverse congruence. On the other hand, if $\rho$ is a locally isomorphic inverse congruence, then $\rho=\gamma$ by (i).

We now describe our main construction. Let $S$ be an inverse semigroup. Let $I$ be a set and let $p: I \times I \rightarrow S$ be a sandwich function. We write the Rees matrix semigroup determined by $S$ and $p$ as $M=M(S, I, p)=I \times S \times I$. Put $R=R M(S, I, p)$ the set of all regular elements of $M$.

Definition. A sandwich function $p: I \times I \rightarrow S$ will be called normal if the following conditions hold:

(S1) $p_{i, i} \in E(S)$ for all $i \in I$.

(S2) $p_{i, j}=\left(p_{j, i}\right)^{-1}$ for all $i, j \in I$.

(S3) $p_{i, j} p_{j, k} \leq p_{i, k}$ for all $i, j, k \in I$.

A Rees matrix semigroup $M=M(S, I, p)$ over an inverse semigroup $S$ is said to be normalised if $p$ is a normal sandwich function.

The above conditions are special cases of conditions appearing in Lemma 2.2 of [27].

It is somewhat surprising that normalised sandwich matrices appear naturally in differential geometry. See [5] for the definitions from the theory of differentiable manifolds, and [19] for some further comments related to this example.

Example. Let $M$ be a topological space. Let $\mathscr{A}=\left\{\phi_{i}: i \in I\right\}$ be a family of homoemorphisms $\phi_{i}: U_{i} \rightarrow V_{i}$ where $U_{i}$ is an open subset of $M$ and $V_{i}$ is an open subset of $\mathbb{R}^{n}$. Let $\Gamma\left(\mathbb{R}^{n}\right)$ be the inverse semigroup of all diffeomorphisms between open subsets of $\mathbb{R}^{n}$. We suppose that $M$ is covered by the $U_{i}$ and that for all $i, j \in I$ we have that $\phi_{i} \phi_{j}^{-1} \in \Gamma\left(\mathbb{R}^{n}\right)$. Thus $\mathscr{A}$ is an atlas compatible with the inverse semigroup $\Gamma\left(\mathbb{R}^{n}\right)$, and consequently defines a smooth manifold on $M$. Define a function $p: I \times I \rightarrow \Gamma\left(\mathbb{R}^{n}\right)$ by $p(i, j)=\phi_{i} \phi_{j}^{-1}$. Then it can be easily checked that $p$ is a normal sandwich function.

Theorem 6.7. Let $M=M(S, I, p)$ be a normalised Rees matrix semigroup, and let $R=R(S, I, p)$ be its semigroup of regular elements. Then

(i) For all $i, j \in I$, we have that $p_{i, i} p_{i, j}=p_{i, j}$ and $p_{i, j} p_{j, j}=p_{i, j}$.

(ii) $(i, s, j) \in E(M)$ if, and only if, $s \leq p_{i, j}$. 
(iii) $R$ is orthodox.

(iv) $R$ is a generalised inverse semigroup.

(v) $(i, s, j) \in R$ if, and only if, $s^{-1} s \leq p_{j, j}$ and $s s^{-1} \leq p_{i, i}$.

(vi) If $(i, s, j) \in R$ then

$$
V(i, s, j)=\left\{\left(k, p_{k, j} s^{-1} p_{i, l}, l\right): s s^{-1} \leq p_{i, l} p_{l, i} \quad \text { and } \quad s^{-1} s \leq p_{j, k} p_{k, j}\right\}
$$

(vii) If $(i, s, j),(k, t, l) \in R$ then

$$
(i, s, j) \mathscr{R}(k, t, l) \quad \text { if, and only if, } \quad i=k \text { and } s \mathscr{R} .
$$

(viii) If $(i, s, j),(k, t, l) \in R$ then

$$
(i, s, j) \mathscr{L}(k, t, l) \text { if, and only if, } j=1 \text { and } s \mathscr{L} t .
$$

(ix) On $R,((i, s, j)(k, t, l)) \in \gamma$ if, and only if, $s=p_{i k} t p_{l j}$ and $t=p_{k i} s p_{j l}$.

Proof. (i) We prove the first equality, the proof of the second is similar. By (S2) and (S3) we have that

$$
p_{i, j}\left(p_{i, j}\right)^{-1}=p_{i, j} p_{j, i} \leq p_{i, j}
$$

The result is now immediate.

(ii) $(i, s, j)$ is an idempotent if, and only if, $s=s p_{j, i} s$. Hence $(i, s, j)$ is an idempotent if, and only if, $s^{-1}=p_{j, i} s p_{j, i}$. Clearly $s p_{j, i}$ is an idempotent and so $s^{-1} \leq p_{j, i}$. Hence by (S2), $s \leq p_{i j}$. Conversely, if $s \leq \dot{p}_{i, j}$ then $s=p_{i, j} s^{-1} s$, so that $s^{-1}=s^{-1} s p_{j, i}$. Thus

$$
s=s s^{-1} s=s\left(s^{-1} s p_{j, i}\right) s=s p_{j, i} s .
$$

(iii) Let $(i, s, j),(k, t, l) \in E(M)$. Then by (ii) above,

$$
s \leq p_{i, j} \quad \text { and } \quad t \leq p_{k, l}
$$

Thus by (S3), we obtain $s p_{j, k} t \leq p_{i, l}$. But then by (ii) again this implies that $(i, s, j)(k, t, l)=\left(i, s p_{j, k} t, l\right)$ is an idempotent.

(iv) $R$ is a locally inverse regular semigroup by Result 1.11, and orthodox by (iii), and so it is a generalised inverse semigroup.

(v) Suppose that $(i, s, j)$ is regular. Then there exists $(k, t, l) \in V(i, s, j)$. This implies that $s=s p_{j, k} t p_{l, i} s$. Now,

$$
p_{j, j} s^{-1} s=p_{j, j} s^{-1} s p_{j, k} t p_{l, i} s=s^{-1} s p_{j, j} p_{j, k} t p_{l, i} s
$$

since $p_{j, j}$ is an idempotent by (S1). But $p_{j, j} p_{j, k}=p_{j, k}$ by (i) above. Thus

$$
p_{j, j} s^{-1} s=s^{-1} s p_{j, k} t p_{l, i} s=s^{-1} s
$$


and so $s^{-1} s \leq p_{j, j}$. Similarly, $s s^{-1} \leq p_{i, i}$. Conversely, suppose that $(i, s, j)$ is such that $s^{-1} s \leq p_{j, j}$ and $s s^{-1} \leq p_{i, i}$. Consider the element $\left(j, s^{-1}, i\right)$. Then

$$
(i, s, j)\left(j, s^{-1}, i\right)(i, s, j)=\left(i, s p_{j,} s^{-1} p_{i, i} s, j\right)
$$

But $s p_{j, j} s^{-1} p_{i, i} s=s$ since $s^{-1} s \leq p_{j, j}$ and $s s^{-1} \leq p_{i, i}$. Thus $(i, s, j)$ is regular.

(vi) Let $(k, t, l) \in V(i, s, j)$. Then $s=s p_{j, k} t p_{l, i} s$ and $t=t p_{l, i} s p_{j, k} t$. Thus

$$
s p_{j, k}=\left(s p_{j, k}\right)\left(t p_{l, i}\right)\left(s p_{j, k}\right) \text { and } t p_{l, i}=\left(t p_{l, i}\right)\left(s p_{j, k}\right)\left(t p_{l, i}\right)
$$

and so $t p_{l, i}=\left(s p_{j, k}\right)^{-1}$. From $s=s p_{j, k} t p_{l, i} s$ we obtain

$$
s^{-1} s \leq p_{j, k} p_{k, j} \text { and } \quad s s^{-1} \leq p_{i, l} p_{l, i} \text {. }
$$

Similarly,

$$
t^{-1} t \leq p_{l, i} p_{i, l}
$$

Thus

$$
t=t p_{l, i}\left(p_{l, i}\right)^{-1}=\left(p_{j, k}\right)^{-1} s^{-1}\left(p_{l, i}\right)^{-1}
$$

and so $t=\left(p_{j, k}\right)^{-1} s^{-1}\left(p_{l, i}\right)^{-1}=p_{k j} s^{-1} p_{i, l}$. The converse is easy to check.

(vii) Let $(i, s, j),(k, t, l) \in R$. Suppose that $i=k$ and that $s \mathscr{R} t$. By definition there exist elements $a$ and $b$ such that $s a=t$ and $t b=s$. By (v) above, we have that $s^{-1} s \leq p_{j, j}$ and $t^{-1} t \leq p_{l, l}$. Thus

$$
s p_{j, j} a=t \text { and } t p_{l, l} b=s
$$

But then

$$
(i, s, j)\left(j, p_{j, j} a p_{l, l}, l\right)=\left(i, s p_{j, j} p_{j, j} a p_{l, l}, l\right)=(i, t, l)
$$

Similarly,

$$
(i, t, l)\left(l, p_{l, l} b p_{j, j}, j\right)=(i, s, j)
$$

Furthermore, by $(v)$, both $\left(j, p_{j, j} a p_{l, l}, l\right)$ and $\left(l, p_{l, l} b p_{j, j}, j\right)$ are regular. Thus $(i, s, j) \mathscr{R}(k, t, l)$. The converse is straightforward to check.

(viii) Similar to the proof of (vii) above.

(ix) Note first that, by (iii), $R$ is orthodox. Suppose that $((i, s, j),(k, t, l)) \in \gamma$. Then by Result 1.8 , we must have that $V((i, s, j))=V((k, t, l))$. A particular inverse of $(i, s, j)$ is the element $\left(j, s^{-1}, i\right)$ by $(v)$. Thus $\left(j, s^{-1}, i\right) \in V((k, t, l))$, and so

$$
t=t p_{l j} s^{-1} p_{i k} t \quad \text { and } \quad s^{-1}=s^{-1} p_{i k} t p_{l j} s^{-1} .
$$


Thus

$$
s=s s^{-1} p_{i k} t p_{l j} s^{-1} s \leq p_{i k} t p_{l j}
$$

and

$$
t^{-1}=t^{-1} t p_{l j} s^{-1} p_{i k} t t^{-1} \leq p_{i j} s^{-1} p_{i k}
$$

so that $t \leq p_{k i} s p_{j i}$. But

$$
p_{k i} s p_{j l} \leq p_{k i}\left(p_{i k} t p_{l j}\right) p_{j l}
$$

thus by (S3), we have that

$$
p_{k i} s p_{j l} \leq p_{k k} t p_{l l}
$$

But by (v), we have that $t^{-1} t \leq p_{l l}, t t^{-1} \leq p_{k k}$, so that $p_{k i} s p_{j l} \leq t$. Thus $t=p_{k i} s p_{j l}$. Similarly, $s=p_{i k} t p_{l j}$.

Conversely, suppose that $t=p_{k i} s p_{j l}$ and $s=p_{i k} t p_{i j}$. We shall show that $V((i, s, j)) \cap V((k, t, l))$ is non-empty. To do this, we show that $\left(j, s^{-1}, i\right) \in V((k, t, l))$. Calculate,

$$
t p_{l j} s^{-1} p_{i k} t=t\left(p_{l j} s^{-1} p_{i k}\right) t=t\left(p_{k i} s p_{j l}\right)^{-1} t=t t^{-1} t=t .
$$

Similarly, $s^{-1}=s^{-1} p_{i k} t p_{l j} s^{-1}$. The result now follows by Result 1.8 .

We now consider the case where $S$ is a monoid with identity $e$. We say that a normalised sandwich function $p: I \times I \rightarrow S$ is pointed if it satisfies:

(S4) For some $1 \in I, p_{1,1}=e$.

Proposition 6.8. Let $R=R M(S, I, p)$ be a pointed normalised Rees matrix semigroup over an inverse semigroup $S$ with identity $e$. Put $\mathrm{e}=(1, e, 1)$. Then

(i) $R=R \mathbf{e}$.

(ii) $S$ is isomorphic to eRe.

Proof. (i) If $(i, s, j) \in R$ then $(i, s, 1),\left(s, s^{-1} s, j\right) \in R$ by Theorem $6.7(v)$. But then

$$
(i, s, j)=(i, s, 1)(1, e, 1)\left(1, s^{-1} s, j\right)
$$

(ii) Define a function $\theta: S \rightarrow \mathrm{e} \operatorname{Re}$ by $\theta(s)=(1, s, 1)$. It is easy to check that $\theta$ is an isomorphism.

From the above result we have a way of constructing a class of generalised inverse enlargements of inverse monoids. 
Lemma 6.9. Let $R$ be a generalised inverse semigroup, and let $e$ be an idempotent of $R$. Suppose that $R=R e R$ and $S=e R e$. Then $S$ is isomorphic to $\gamma(e)(R / \gamma) \gamma(e)$ and $R / \gamma=(R / \gamma) \gamma(e)(R / \gamma)$.

Proof. Observe first, that if $\theta: R \rightarrow T$ is any surjective homomorphism, then $T=T \theta(e) T$ and $\theta(e R e)=\theta(e) T \theta(e)$. By Theorem 6.6, $\gamma^{\natural}$ is a local isomorphism, since $S$ is a generalised inverse semigroup. This implies that $e R e$ and $\gamma(e)(R / \gamma) \gamma(e)$ are isomorphic. The result is now immediate.

By Theorem 6.7(iv), Proposition 6.8 and Lemma 6.9 we now have the following way of constructing a class of inverse enlargements of an inverse monoid $S$.

Theorem 6.10. Let $S$ be an inverse semigroup with identity $e$ and let $R=R M(S, I, p)$ be a pointed normalised Rees matrix semigroup over $S$. Put $\mathrm{e}=(1, e, 1)$. Then $S$ is isomorphic to $\gamma(\mathbf{e})(R / \gamma) \gamma(\mathbf{e})$ and $R / \gamma=(R / \gamma) \gamma(\mathbf{e})(R / \gamma)$. Thus $R / \gamma$ is an inverse enlargement of a local submonoid isomorphic to $S$.

That every inverse enlargement of $S$ is obtained as above is the substance of the following result.

Theorem 6.11. Let $S$ be an inverse monoid with identity $e$ and let $T$ be an inverse enlargement of $S$. Then there exists a pointed normalised Rees matrix semigroup $R=R M(S, I, p)$ over $S$ such that $T$ is isomorphic to $R / \gamma$.

Proof. We follow the procedure of Theorem 4.2. Put $I=E(T)$. For each idempotent $i \in E(T)$, pick an element $x_{i} \in T$ such that $\left(x_{i}\right)^{-1} x_{i} \leq e$ and $x_{i}\left(x_{i}\right)^{-1}=i$. If $i=e$, then we choose $x_{i}=e$. If $i, j \in I$, then define

$$
p_{i, j}=\left(x_{i}\right)^{-1} x_{j}
$$

It is clear that $p_{i, j} \in S$. It is easy to check that $p$ is a pointed normalised sandwich function. We now prove the remainder of the theorem. By Theorem 4.2, the function $\theta: R M(S, I, p) \rightarrow T$ defined by $\theta(i, s, j)=x_{i} s\left(x_{j}\right)^{-1}$ is a surjective local isomorphism. By Theorem 6.7, $R M(S, I, p)$ is a generalised inverse semigroup, and by Theorem $6.6, \gamma$ is the unique locally isomorphic inverse congruence on $\operatorname{RM}(S, I, p)$. Thus $\operatorname{ker} \theta=\gamma$ and so $T$ is isomorphic to $\operatorname{RM}(S, I, p) / \gamma$. (The fact that $\operatorname{ker} \theta=\gamma$ can also be seen directly for:

$$
\theta(i, s, j)=\theta(k, t, l) \Leftrightarrow x_{i} s x_{j}^{-1}=x_{k} t x_{i}^{-1}
$$

By Theorem 6.7(v), $x_{i} s x_{j}^{-1}=x_{k} t x_{l}^{-1}$ if, and only if,

$$
s=\left(x_{i}^{-1} x_{k}\right) t\left(x_{i}^{-1} x_{j}\right) \text { and } t=\left(x_{k}^{-1} x_{i}\right) s\left(x_{j}^{-1} x_{i}\right) .
$$


But by Theorem 6.7(ix), these equations hold if, and only if, $(i, s, j) \gamma(k, t, l)$.)

\section{Ordered groupoid enlargements}

In this section, we describe how enlargements may be interpreted using ordered groupoids.

A groupoid $G$ is a category in which every element is an isomorphism. If $x \in G$ then $\mathbf{d}(x)$ and $\mathbf{r}(x)$ are the right and left identities of $x$ respectively. The set of all identities of $G$ is denoted by $G_{o}$. The product $x y$ is defined precisely when $\mathbf{d}(x)=\mathbf{r}(y)$. The inverse of $x$ is denoted by $x^{-1}$. Clearly, $x^{-1} x=\mathrm{d}(x)$ and $x x^{-1}=\mathrm{r}(x)$. An ordered groupoid is a groupoid equipped with a partial order $\leq$ such that the following axioms hold:

(G) $x \leq y$ implies that $x^{-1} \leq y^{-1}$.

(OC3) $x \leq y$ and $x^{\prime} \leq y^{\prime}$ and $x x^{\prime}$ and $y y^{\prime}$ defined implies that $x x^{\prime} \leq y y^{\prime}$.

(OC8) (i) If $e$ is an identity such that $e \leq \mathrm{d}(x)$ then there exists a unique element, denoted $(x \mid e)$, called the restriction of $x$ to $e$, such that $(x \mid e) \leq x$ and $\mathrm{d}(x \mid e)=e$.

(OC8) (ii) If $e$ is an identity such that $e \leq \mathbf{r}(x)$ then there exists a unique element, denoted $(e \mid x)$, called the corestriction of $x$ to $e$, such that $(e \mid x) \leq x$ and $\operatorname{r}(e \mid x)=e$.

An ordered groupoid is said to be inductive if the set of identities forms a meet semilattice under the induced order.

If $G$ is an ordered groupoid and $H$ a subset of $G$ then we say that $H$ is an ordered subgroupoid of $G$ if it is a subgroupoid of $G$ and an ordered groupoid with respect to the induced order. This is equivalent to the condition that $H$ be a subgroupoid of $G$ and that if $x \in H$ and $e \in H_{o}$ and $e \leq \mathrm{d}(x)$ (resp. $e \leq \mathrm{r}(x)$ ) then $(x \mid e) \in H$ (resp. $(e \mid x) \in H)$.

We now recall a construction due to Nambooripad [36]. Let $S$ be a regular semigroup and let $\mathbf{G}(S)$ be the set of all inverse pairs in $S$. On $\mathbf{G}(S)$ define a partial product in which $\left(x, x^{\prime}\right)\left(y, y^{\prime}\right)$ is defined to be $\left(x y, y^{\prime} x^{\prime}\right)$ if $x^{\prime} x=y y^{\prime}$, and undefined otherwise. It is easy to check that under the given conditions $\left(x y, y^{\prime} x^{\prime}\right)$ is an inverse pair. Define a partial order on $\mathbf{G}(S)$ by $\left(x, x^{\prime}\right) \leq\left(y, y^{\prime}\right)$ if $x x^{\prime} \leq y y^{\prime}, x=x x^{\prime} y$ and $x^{\prime}=y^{\prime} x x^{\prime}$; observe that if $\left(x, x^{\prime}\right) \leq\left(y, y^{\prime}\right)$ then $x^{\prime} x \leq y^{\prime} y$ holds automatically. With respect to the partial product and order just defined it can be shown that $\mathbf{G}(S)$ is an ordered groupoid. The identities of $\mathbf{G}(S)$ are the elements of the form $(e, e)$ where $e$ is an idempotent of $S$, and

$$
\left(x, x^{\prime}\right)^{-1}=\left(x^{\prime}, x\right), \mathrm{d}\left(x, x^{\prime}\right)=\left(x^{\prime} x, x^{\prime} x\right) \text { and } \mathbf{r}\left(x, x^{\prime}\right)=\left(x x^{\prime}, x x^{\prime}\right)
$$

The restrictions and corestrictions are given by

$$
\left(\left(x, x^{\prime}\right) \mid(e, e)\right)=\left(x e, e x^{\prime}\right) \text { and }\left((e, e) \mid\left(x, x^{\prime}\right)\right)=\left(e x, x^{\prime} e\right)
$$

where $(e, e) \leq \mathrm{d}\left(x, x^{\prime}\right)$ and $(e, e) \leq \mathbf{r}\left(x, x^{\prime}\right)$ respectively

The following is immediate from Result 1.2. 
Lemma 7.1. If $\left(x, x^{\prime}\right) \leq\left(y, y^{\prime}\right)$ in $\mathbf{G}(S)$ then $x \leq y$ and $x^{\prime} \leq y^{\prime}$ in $S$.

Let $G$ be a subgroupoid of a groupoid $H$. Then $G$ is said to be a full subgroupoid if $x \in H$ and $\mathrm{d}(x), \mathbf{r}(x) \in G$ imply that $x \in G$. $G$ is said to be dense in $H$ if for every identity $e$ in $H$ there is an element $x \in H$ such that $\mathbf{r}(x)=\mathbf{e}$ and $\mathrm{d}(x) \in G$. Now let $G$ be an ordered subgroupoid of $H$. We say that $H$ is an enlargement of $G$ if, and only if, the following axioms hold:

(GE1) $G$ is an order ideal of $H$,

(GE2) $G$ is a full subgroupoid of $H$,

(GE3) $G$ is a dense subgroupoid of $H$.

Proposition 7.2. Let $S$ be a regular subsemigroup of $T$. Then $T$ is an enlargement of $S$ if, and only if, $\mathbf{G}(T)$ is an enlargement of $\mathbf{G}(S)$.

Proof. Let $T$ be an enlargement of $S$. We first show that $\mathbf{G}(S)$ is an ordered subgroupoid of $\mathbf{G}(T)$. Clearly, $\mathbf{G}(S)$ is a subgroupoid of $\mathbf{G}(T)$. Let $\left(x, x^{\prime}\right),(e, e) \in \mathbf{G}(S)$ where $(e, e) \leq \mathrm{d}\left(x, x^{\prime}\right)$ in $\mathbf{G}(T)$. Then $x, x^{\prime}, e \in S$ and so $\left(\left(x, x^{\prime}\right) \mid(e, e)\right)=\left(x e, e x^{\prime}\right) \in \mathbf{G}(S)$. A similar argument for corestrictions shows that $\mathbf{G}(S)$ is an ordered subgroupoid of $\mathbf{G}(T)$.

(GE1) holds: let $\left(x, x^{\prime}\right) \leq\left(y, y^{\prime}\right) \in \mathbf{G}(S)$. Then by Lemma 7.1 , we have that $x \leq y$ and $x^{\prime} \leq y^{\prime}$, so that $x, x^{\prime} \in S$ since $S$ is an order ideal of $T$. Thus $\left(x, x^{\prime}\right) \in \mathbf{G}(S)$. (GE2) holds: let $\left(x, x^{\prime}\right) \in \mathbf{G}(T)$ such that $\left(x^{\prime} x, x^{\prime} x\right),\left(x x^{\prime}, x x^{\prime}\right) \in \mathbf{G}(S)$. Then $x^{\prime} x, x x^{\prime} \in S$ so that $x, x^{\prime} \in S$. Thus $\left(x, x^{\prime}\right) \in \mathbf{G}(S)$. (GE3) holds: let $(e, e)$ be an identity of $\mathbf{G}(T)$. Since $T$ is an enlargement of $S$ there exists $x \in T$ and $x^{\prime} \in V(x)$ such that $x x^{\prime}=e$ and $x^{\prime} x \in E(S)$. Thus $\left(x, x^{\prime}\right) \in \mathbf{G}(T), \mathbf{r}\left(x, x^{\prime}\right)=(e, e)$ and $\mathbf{d}\left(x, x^{\prime}\right) \in \mathbf{G}(S)_{o}$.

Conversely, suppose that $\mathbf{G}(T)$ is an enlargement of $\mathbf{G}(S)$. (E1) holds: let $y \leq x \in S$ and let $x^{\prime} \in V(x) \cap S$. By Result 1.2(ii), there exists $e \in E\left(R_{y}\right)$ such that $e \leq x x^{\prime}$ and $y=e x$. Put $y^{\prime}=x^{\prime} e$. It is easy to check that $y^{\prime} \in V(y)$. Thus $\left(x, x^{\prime}\right) \in \mathbf{G}(S)$ and $\left(y, y^{\prime}\right) \in \mathbf{G}(T)$. Now observe that

$$
y y^{\prime}=e \leq x x^{\prime}, y=y y^{\prime} x \quad \text { and } \quad y^{\prime}=x^{\prime} y y^{\prime} .
$$

Thus $\left(y, y^{\prime}\right) \leq\left(x, x^{\prime}\right)$. Hence $\left(y, y^{\prime}\right) \in \mathbf{G}(S)$, and so $y \in S$. (E2) holds: let $x \in T$ and $x^{\prime} \in V(x)$ such that $x^{\prime} x, x x^{\prime} \in E(S)$. Then $\left(x, x^{\prime}\right) \in \mathbf{G}(T)$ and $\mathrm{d}\left(x, x^{\prime}\right), \mathrm{r}\left(x, x^{\prime}\right) \in \mathbf{G}(S)$, so that $\left(x, x^{\prime}\right) \in \mathbf{G}(S)$ by (GE2). Hence $x \in S$. Finally, (E3) holds: let $e \in E(T)$. Then $(e, e)$ is an identity of $\mathbf{G}(T)$. Thus by (GE3), there exists $\left(x, x^{\prime}\right) \in \mathbf{G}(T)$ such that $\mathbf{r}\left(x, x^{\prime}\right)=(e, e)$ and $\mathbf{d}\left(x, x^{\prime}\right) \in \mathbf{G}(S)$. It follows that $x x^{\prime}=e$ and $x^{\prime} x \in E(S)$.

We conclude this section with some comments on the origins of our notion of an 'enlargement'. The definition of enlargement for ordered groupoids is due to Ehresmann [7], although (GE1) is not explicitly mentioned. The semigroup theoretic definition of enlargement is essentially due to McAlister [24], where conditions (E1) and (E2) are the basis for what McAlister there calls 'heavy' subsemigroups. Condition (E3) is mentioned in passing at the end of this paper. The connection between 
conditions (E1) and (E2) and quasi-ideals is spelt out in [29]. Our real contribution lies in recognising that it is the combination of these three conditions which is important.

\section{Applications}

In this section, we shall consider some applications of the theory of enlargements to regular semigroups.

\section{§1. Completely (0-) simple semigroups}

Proposition 8.1. A regular semigroup $T$ is completely (0-)simple if, and only if, it is $a(0-)$ enlargement of a group (resp. group with zero) $S$.

Proof. We shall consider the completely simple case; the completely 0 -simple case is proved similarly. Suppose that $S$ is a group and $T$ is an enlargement of $S$. Since $S$ is simple $T$ is simple by Theorem 3.4(iii). But $S$ is an order ideal of $T$ and so every primitive idempotent of $S$ is a primitive idempotent of $T$. By Corollary III.3.4 of [13], it follows that $T$ is completely simple. Conversely, if $T$ is completely simple then for any idempotent $e, S=e T e$ is a group. Since $T$ is bisimple, $T$ is an enlargement of $S$.

The following results are well-known but they are immediate consequences of Theorem 5.4, Theorem 6.4 and Proposition 8.1. The latter result was proved by Preston [40] and the former is a special case of results discussed by Howie [13].

Corollary 8.2. (i) Let $S$ be a completely 0 -simple semigroup and $e$ a non-zero idempotent. Then the lattice of idempotent separating congruences on $S$ is isomorphic to the lattice of idempotent separating congruences on the group with zero eSe. In particular, this lattice is isomorphic to the lattice of normal subgroups of the group of eSe.

(ii) Let $S$ be a Brandt semigroup and e a non-zero idempotent. Then the lattice of all congruences on $S$ is isomorphic to the lattice of all congruences on the group with zero eSe. In particular, every proper congruence on $S$ is idempotent separating.

\section{§2. (0-)bisimple regular semigroups}

Let $S$ be a 0 -bisimple regular semigroup and $e \in S$ a non-zero idempotent. Then $S$ is a 0-enlargement of $e S e$, and $e S e$ is a 0 -bisimple regular monoid. The lattice of idempotent separating congruences on $S$ is isomorphic to the lattice of idempotent separating congruences on $e S e$. Thus questions about such congruences need only be studied in the monoid case. Compare with [35]. If $S$ is also inverse then $e S e$ will likewise be inverse. It follows that the lattice of all congruences on $S$ is isomorphic to the lattice of all congruences on $e S e$. Thus in the inverse case, questions about congruences need only be studied in the monoid case. Compare with [39].

There are a number of structure theorems for (0-)bisimple inverse semigroups [23], [41], [42]. We now derive another such result. Let $S$ be a 0 -bisimple inverse semigroup 
and $e$ a non-zero idempotent. Thus $S$ is an enlargement of the inverse monoid $e S e$. By Theorem 6.11, there is a pointed, normalised sandwich function $p: I \times I \rightarrow e S e$ such that $S$ is isomorphic to $R M(e S e, I, p) / \gamma$. The function $\theta: R M(e S e, I, p) \rightarrow S$ is given by $\theta(i, s, j)=x_{i} s x_{j}^{-1}$, and $\theta(i, s, j)=\theta(k, t, l)$ iff $s=p_{i, k} t p_{l j}$ and $t=p_{k, i} s p_{j, l}$. Thus

$$
\theta(i, s, j)=\theta(k, 0, l) \text { iff } s=p_{i, k} 0 p_{l, j} \text { and } 0=p_{k, i} s p_{j, l},
$$

and so $\theta(i, s, j)=\theta(k, 0, l)$ iff $s=0$. Thus the ideal

$$
J=\{(i, 0, j): i, j \in I\}
$$

is a $\gamma$-class. Form the semigroup $R M^{0}(e S e, I, p)$. Since it is a Rees quotient of $R M(e S e, I, p)$ it is still a generalised inverse semigroup. It is clear that $S$ is isomorphic to $R M^{0}(e S e, I, p) / \gamma$, where $\gamma$ is the minimum inverse congruence on $R M^{0}(e S e, I, p)$. We now have the following alternative structure theorem for 0 -bisimple inverse semigroups. It can be regarded as a generalisation of the structure of Brandt semigroups.

Theorem 8.3. Every 0-bisimple inverse semigroup is isomorphic to a semigroup of the form $R^{0}(S, I, p) / \gamma$ where $S$ is a 0-bisimple inverse monoid and $p: I \times I \rightarrow S$ is a pointed, normalised sandwich function.

\section{§3. Locally inverse semigroups}

Let $S$ be a locally inverse regular semigroup. By Theorem 5.6 of [30], $S$ can be embedded as a regular quasi-ideal in a regular, locally inverse semigroup $U$ possessing an idempotent $e$ such that $U=U e U$. It is convenient to regard $S$ as a regular subsemigroup of $U$. Clearly, $U$ is an enlargement of the inverse semigroup eUe. Put $T=\mathscr{E}_{U}(S)$ and $I=\mathscr{E}_{U}(S) \cap e U e$. Then by Proposition 2.4,T is an enlargement of both $S$ and the inverse semigroup 1 . Note that $I$ consists of those elements of $e U e$ which are $\mathscr{D}$-related to an element of $S$.

The structures of the locally inverse semigroup $S$ and the inverse semigroup $I$ are very closely related: they have isomorphic lattices of idempotent separating congruences (by Theorem 5.4), the posets $S / \mathscr{F}$ and $I / \mathscr{J}$ are isomorphic (by Theorem 3.3(ii)) and the lattices of two-sided ideals of $S$ and $I$ are isomorphic (by Theorem 3.3(i)). Furthermore, $S$ is combinatorial (simple, bisimple, fundamental) precisely when $I$ is (by Theorem 3.4 and Corollary 5.5).

Similar results hold for regular semigroups $S$ which are locally $\mathscr{L}$-unipotent, locally orthodox, locally $E$-solid, locally a union of groups with $S / \mathscr{F}$ a semilattice, and locally testable with $S / \mathscr{J}$ a semilattice in terms of $\mathscr{L}$-unipotent, orthodox, $E$-solid, unions of groups, and semilattices.

\section{\$4. Inverse enlargements of Munn semigroups}

Let $S$ be a fundamental inverse semigroup. By Corollary 5.5, any inverse enlargement $T$ of $S$ is also fundamental. The most natural class of fundamental inverse semigroups are the Munn semigroups. In the next result, we characterise the enlargements of Munn semigroups $T_{E}$ in terms of the semilattice $E$. 
Theorem 8.4. (i) Let $E$ and $F$ be meet semilattices with $E$ an order ideal of $F$. Suppose that every principal order ideal of $F$ is isomorphic to a principal order ideal of $E$. Then $T_{F}$ is an enlargement of $T_{E}$.

(ii) Every enlargement $T$ of a Munn semigroup $T_{E}$ is isomorphic to a Munn semigroup $T_{F}$, where $E$ is an order ideal of $F$ and every principal order ideal of $F$ is isomorphic to an order ideal of $E$.

Proof. (i) Straightforward.

(ii) Let $S=T_{E}$ and let $T$ be an enlargement of $S . T$ is fundamental, so that if $E(T)=F$ we can assume, by suitable relabelling of elements of $T$, that $T$ is a full inverse subsemigroup of $T_{F}$. Thus

$$
T_{E} \subseteq T \subseteq T_{F}
$$

We may also assume that $E \subseteq F$ is an order ideal. Let $\alpha \in T_{F}$. Both $\alpha^{-1} \alpha$ and $\alpha \alpha^{-1}$ are identity maps on principal order ideals of $F$. Thus both idempotents belong to $T$, since $T$ is a full subsemigroup of $T_{F}$. But $T$ is an enlargement of $T_{E}$, so that there exist elements $\beta, \gamma \in T$ such that

$$
\beta \beta^{-1}=\alpha \alpha^{-1} \text { and } \gamma \gamma^{-1}=\alpha^{-1} \alpha \text { and } \beta^{-1} \beta, \gamma^{-1} \gamma \in T_{E}
$$

Clearly, $\delta=\beta^{-1} \alpha \gamma \in T_{E}$, and so $\alpha=\beta \delta \gamma^{-1} \in T$. Hence $T=T_{F}$.

We showed in [18], that there is a very close relationship between a class of enlargements of an inverse semigroup $S$ and $E$-unitary covers of $S$ over semilattices. We shall consider one aspect of this relationship with regard to Munn semigroups. We first need some definitions.

A pair of elements $x$ and $y$ in an inverse semigroup $S$ are said to be compatible, written $x \sim y$, if both $x y^{-1}$ and $x^{-1} y$ are idempotents. A subset $A$ of $S$ is said to be compatible if the elements of $A$ are pairwise compatible. A permissible subset of $S$ is a compatible order ideal. A permissible subset $A$ of $S$ is said to be invertible if $A A^{-1}=A^{-1} A=E(S)$. The set of all invertible, permissible subsets of $S$ is denoted $\Sigma(S)$, and forms a group. The following definition is due to McAlister, see [18] for more details: an inverse semigroup $S$ is said to be almost factorisable if for each $x \in S$ there exists $A \in \Sigma(S)$ such that $x \in A$.

Theorem 8.5. The almost factorisable enlargements of $T_{E}$ are all isomorphic to the semigroups $T_{F}$, where $E$ and $F$ satisfy the following conditions:

(ME1) $E$ is an order ideal of $F$,

(ME2) every principal order ideal of $F$ is isomorphic to a principal order ideal of $E$,

(ME3) every isomorphism between principal order ideals of $F$ is induced by an automorphism of $F$.

Proof. We show first that every element of $\Sigma\left(T_{F}\right)$ determines, and is determined 
by, an element of $\operatorname{Aut}(F)$. More precisely, define functions $\mathscr{F}: \Sigma\left(T_{F}\right) \rightarrow \operatorname{Aut}(F)$ and $\mathscr{G}: \operatorname{Aut}(F) \rightarrow \Sigma\left(T_{F}\right)$ as follows:

$$
\mathscr{F}(A)=\cup A \text { and } \mathscr{G}(\theta)=\{(\theta \mid[e]): e \in F\},
$$

where $[e]$ is the principal order ideal determined by $e$ in $F$. We show first that these functions are well-defined. We begin with $\mathscr{F}$. Let $A \in \Sigma\left(T_{F}\right)$. Then $A$ is an invertible, permissible subset of $T_{F}$. Thus $\mathscr{F}(A)$ is, a priori, a relation on $F$. It is easy to check that if $\alpha, \beta \in T_{F}$ then $\alpha \sim \beta$ if, and only if, $\alpha \cup \beta$ is an isomorphism from the order ideal $\operatorname{dom}(\alpha) \cup \operatorname{dom}(\beta)$ to the order ideal $\operatorname{ran}(\alpha) \cup \operatorname{ran}(\beta)$. It follows from this that $\mathscr{F}(A)$ is a partial isomorphism of $F$ with domain and range order ideals of $F$. That it is an automorphism is immediate from the fact that $A$ is invertible. Next we show that $\mathscr{G}(\theta)$ is well-defined. It is easy to see that $\mathscr{G}(\theta)$ is a permissible subset of $T_{F}$ since any two partial functions which have the same extension are compatible. $\mathscr{G}(\theta)$ is invertible, since $\theta$ is an automorphism. It is clear that $\mathscr{G} \mathscr{F}$ and $\mathscr{F} \mathscr{G}$ are the identity maps. Finally, both $\mathscr{F}$ and $\mathscr{G}$ are group homomorphisms. To show that $\mathscr{F}$ is a homomorphism, we have to show that for all $A, B \in \Sigma\left(T_{F}\right)$, we have that $(\cup A)(\cup B)=\cup(A B)$. Let $x \in F$. Then $((\cup A)(\cup B)) x=(\cup A)((\cup B) x)$. Let $\beta \in B$ such that $x \in \operatorname{dom} \beta$. Then $(\cup B) x=\beta(x)$. Let $\alpha \in \cup A$ such that $\beta(x) \in d o m \alpha$. Thus $(\cup A)(\cup B) x=\alpha(\beta(x))$. But $\alpha \in A$ and $\beta \in B$ implies that $\alpha \beta \in A B$. Thus $\cup(A B) x=\alpha(\beta(x))$. From these results, it is now clear that $T_{F}$ is almost factorisable if, and only if, every isomorphism between principal order ideals is induced by an automorphism of $F$. The result now follows by Theorem 8.4.

In view of Theorem 8.5, the following is now a natural question:

Semilattice Embedding Problem. Given any finite semilattice $E$ does there exist a finite semilattice $F$ such that:

(ME1) $E$ is an order ideal of $F$,

(ME2) every principal order ideal of $F$ is isomorphic to a principal order ideal of $E$,

(ME3) every isomorphism between principal order ideals of $F$ is induced by an automorphism of $F$ ?

Remarks. (i) In Henckell and Rhodes [10], the problem is posed of whether every finite inverse monoid has a finite $F$-inverse cover. From [18], this is equivalent to the question of whether every finite inverse monoid has a finite almost factorisable enlargement. A positive answer to Henckell and Rhodes' problem would therefore yield a positive solution to the Semilattice Embedding Problem.

(ii) Observe that to solve the Semilattice Embedding Problem it is enough to find a semilattice $F$ satisfying condtions (ME1) and (ME3), for the set $F^{\prime}=\operatorname{Aut}(F) E$ is a subsemilattice of $F$ containing $E$ satisfying all three conditions.

(iii) The Semilattice Embedding Problem is a refinement of a question stated and solved by McAlister; Theorem 4.2 of [22] shows that every semilattice $E$ can be embedded in a poset $F$ satisfying (ME1) and (ME3).

(iv) Corollary 4.4 of [25], shows that the Semilattice Embedding Problem can be 
solved if we waive the cardinality restriction. A direct proof of this result is provided by Meakin and Pastijn in [33].

\section{§5. Ordered groupoid enlargements of inverse semigroups and the structure of E-unitary semigroups}

We have seen in Proposition 7.2, that if $S$ is a regular subsemigroup of $T$ then $T$ is an enlargement of $S$ if, and only if, $G(T)$ is an enlargement of $G(S)$. It is of course quite possible, though at first sight apparently rather unmotivated, to consider ordered groupoid enlargements of $\mathbf{G}(S)$ which are not of the form $\mathbf{G}(T)$ (for some regular semigroup $T$ containing $S$ ). We say that an ordered groupoid $G$ is an ordered groupoid enlargement of a regular semigroup $S$ if $G$ is an enlargement of $\mathbf{G}(S)$. It is a curious fact, that some results on inverse semigroups $S$ can be described in terms of ordered groupoid enlargements of $S$. We shall show specifically how the structure theory of $E$ unitary semigroups can, in this way, be regarded as another application of enlargements. We first recall some terminology from the theory of ordered groupoids.

A functor between ordered groupoids is said to be ordered if it is isotone. An ordered functor between inductive groupoids is said to be inductive if it preserves the meet operation on the semilattice of identities. An ordered functor $\theta: G \rightarrow H$ is said to reflect partial orders if, whenever $\theta(x) \leq \theta(y)$, there exists an element $x^{\prime}$ in $G$ such that $\theta\left(x^{\prime}\right)=\theta(x)$ and $x^{\prime} \leq y$. An ordered functor $\imath: G \rightarrow H$ which is injective and reflects partial orders is called an embedding. An ordered functor $\imath: G \rightarrow H$ is an embedding iff $l(G)$ is an ordered subgroupoid of $H$.

If $e$ is an identity in a groupoid $G$ then the set $G_{e}$ of all elements $x$ of $G$ such that $\mathrm{d}(x)=e$ is called the star of $G$ at $e$. A functor $\theta: G \rightarrow H$ induces a function $\theta_{e}: G_{e} \rightarrow H_{O(e)}$ for every identity $e$ in $G$. An ordered functor $\theta$ is said to be star injective (resp. star surjective) if all the functions $\theta_{e}$ are injective (resp. surjective) for all identities $e$ of $G$. An ordered functor which is both star injective and star surjective is said to be an ordered covering functor.

Next we observe that ordered groupoid enlargements $G$ of inductive groupoids are always principally inductive; this means that the set $[e]$, where $e$ is an identity of $G$, is always a meet semilattice under the induced order. For a proof see Theorem 5.2 of [16].

Let $G$ be principally inductive. Define a relaton $\sigma$ on $G$ by $(x, y) \in \sigma$ iff there is $z \in G$ such that $z \leq x$ and $z \leq y$. (It can be shown that $\sigma$ is an 'ordered congruence' on $G$ and that $G / \sigma$ is a groupoid which is totally unordered [16]). In the case where $G$ is an inductive groupoid $\sigma$ is just the minimum group congruence.

When $S$ is an inverse semigroup $\mathbf{G}(S)$ is isomorphic to the set $S$ equipped with the trace product and its natural partial order and is an inductive groupoid. If $\theta: S \rightarrow T$ is a homomorphism between inverse semigroups $S$ and $T$ then $\mathbf{G}(\theta)$ is an inductive functor from $\mathbf{G}(S)$ to $\mathbf{G}(T)$. Let $\theta: S \rightarrow T$ be a homomorphism between inverse semigroups. Then $\theta$ is said to be $\mathscr{L}$-injective (resp. $\mathscr{L}$-surjective) if its restriction to all corresponding $\mathscr{L}$-classes is injective (resp. surjective). $\theta$ is said to be $\mathscr{L}$-bijective if it is $\mathscr{L}$-injective and $\mathscr{L}$-surjective. $\mathscr{L}$-injective homomorphisms are often called idempotent pure homomorphisms. The inductive functor $\mathbf{G}(\theta)$ is star injective (resp. star surjective) iff $\theta$ is $\mathscr{L}$-injective (resp. $\mathscr{L}$-surjective). 
The P-Theorem (see Corollary VII.1.14 of [39]) is equivalent to the following result, whose proof we sketch. The detailed proof is given in Theorem 4.10 of [14].

Theorem 8.6. Let $S$ be an inductive groupoid. Then the following are equivalent:

(i) $S$ is E-unitary.

(ii) $S$ has a principally inductive enlargement $T$ such that $\sigma(T) \cap(S \times S)=\sigma(S)$ and $\sigma^{\natural}: T \rightarrow T / \sigma$ is an ordered covering functor.

Proof. Throughout this proof we write $S$ instead of $\mathbf{G}(S)$ etc.

(i) $\Rightarrow$ (ii). Let $S$ be $E$-unitary. By the $P$-Theorem there is a McAlister triple $(G, X, Y)$ such that $S$ is isomorphic to the $P$-semigroup $P=P(G, X, Y)$. To ease notation we shall assume that $P=S$. The ordered groupoid $T$ is defined to be the semi-direct product groupoid $X \times G$ where the partial product $(x, g)(y, h)=(x, g h)$ if $x=g y$ and is undefined otherwise, and the order on $T$ is just the cartesian product order. It is straightforward to check that $T$ is an ordered groupoid and an enlargement of $S$. Next $(x, g) \sigma(y, h)$ iff $g=h$ : the only direction which needs some comment is $g=h$ implies $(x, g) \sigma(y, h)$. This follows from the fact that $(X, \leq)$ is lower directed (Lemma, VII.1.3 of [39]). Thus $\sigma(T) \cap(S \times S)=\sigma(S)$. It is straightforward to check that $\sigma^{\natural}$ is an ordered covering functor.

(ii) $\Rightarrow$ (i). The conditions imply that $\sigma(S)$ is idempotent pure, from which it follows that $S$ is $E$-unitary.

The case where the enlargement obtained above is inductive leads to the following result. For a proof see Proposition 3.4 of [18].

Theorem 8.7. Let $S$ be an inverse semigroup. Then the following are equivalent:

(i) $S$ is E-unitary over a semilattice.

(ii) $S$ has an inverse enlargement $T$ such that $\sigma(T) \cap(S \times S)=\sigma(S)$ and $\sigma(T)^{\natural}$ : $T \rightarrow T / \sigma$ is $\mathscr{L}$-bijective.

In proving the above two theorems we made use of the $P$-Theorem. This is itself a special case of a much more general result called the Maximum Enlargement Theorem (see [15]), which we state below for completeness.

Theorem 8.8. Let $\theta: H \rightarrow K$ be an ordered, star injective functor between ordered groupoids $H$ and $K$. Then there exists an ordered groupoid $G$ and an ordered covering functor $\theta^{\prime}: G \rightarrow K$, and an embedding $\imath: H \rightarrow G$ such that $G$ is an enlargement of $l(H)$ and $\theta_{l}^{\prime}=\theta$. Furthermore, if $\theta$ is surjective then $\theta^{\prime}$ is surjective.

Acknowledgements. Part of the work contained in this paper was carried out whilst the author was visiting Dr David Easdown at the University of Sydney, Australia under an Australian Research Council grant. I would also like to thank Dr Tom Hall of Monash University and Dr Peter Trotter of the University of Tasmania for conversations on parts of this paper, Dr Sunil Talwar of York University for an informative e-mail exchange on the Morita theory of semigroups, and Dr John Hickey of Glasgow University for the references to mid-identities. 


\section{REFERENCES}

1. D. Allen, A generalisation of the Rees theorem to a class of regular semigroups, Semigroup Forum 2 (1971), 321-331.

2. T. S. BLYTH and J. B. Hickey, RP-dominated regular semigroups, Proc. Roy. Soc. Edinburgh 99A (1984), 185-191.

3. T. S. BLYTH and R. MCFADDEN, Naturally ordered regular semigroups with a greatest idempotent, Proc. Roy. Soc. Edinburgh 91A (1981), 107-122.

4. T. S. BLYTH and R. MCFADDEN, On the construction of a class of regular semigroups, $J$. Algebra 81 (1983), 1-22.

5. W. M. Воотнву, An introduction to differential manifolds and Riemannian geometry (Academic Press, 1986).

6. P. V. A. DA Silva, Contributions to combinatorial semigroup theory (Ph.D. Thesis, University of Glasgow, 1991).

7. C. Ehresmann, Oeuvres complètes et commentées (ed. A. C. Ehresmann, Suppl. Cahiers Topologies Géom. Differentielle, Amiens, 1980-1984).

8. T. E. Hall, On regular semigroups, J. Algebra 24 (1973), 1-24.

9. T. E. Hall, Some properties of local subsemigroups inherited by larger subsemigroups, Semigroup Forum 25 (1982), 35-49.

10. K. HenCKell and J. Rhodes, The theorem of Knast, the $P G=B G$ and Type-II conjectures, in Monoids and semigroups with applications (ed. J. Rhodes, World Scientific, 1991).

11. J. B. Hickey, Semigroups under a sandwich operation, Proc. Edinburgh Math. Soc. 26 (1983), 371-382.

12. E. Hotzel, Dual D-operands and the Rees theorem, in Colloquia Mathematica Societatis Janos Bolyai 20, Algebraic theory of semigroups, 247-275.

13. J. M. HowIE, An introduction to semigroup theory (Academic Press, 1976).

14. M. V. Lawson, The geometric theory of inverse semigroups I: $E$-unitary semigroups, $J$. Pure Appl. Algebra 67 (1990), 151-177.

15. M. V. LAwsON, The geometric theory of inverse semigroups II: $E$-unitary covers of inverse semigroups, J. Pure Appl. Algebra 83 (1992), 121-139.

16. M. V. LAwson, The geometric theory of inverse semigroups III: idempotent pure coextensions (U.C.N.W. Maths Preprint 91.23).

17. M. V. Lawson, An equivalence theorem for inverse semigroups, Semigroup Forum 47 (1993), 7-14.

18. M. V. LAwSON, Almost factorisable inverse semigroups. Glasgow Math. J. 35 (1993), 97-1 11.

19. M. V. LAWSON, Inverse semigroup enlargements of inverse monoids (U.C.N.W. Maths Preprint 92.17).

20. M. Loganathan and V. M. Chandrasekaran, Regular semigroups with a split map, Semigroup Forum 44 (1992), 199-212.

21. S. MaCLANE, Categories for the working mathematician (Springer-Verlag, 1971).

22. D. B. MCAlister, Groups, semilattices and inverse semigroups II, Trans. Amer. Math. Soc. 196 (1974), 351-370.

23. D. B. McAlister, 0-bisimple inverse semigroups, Proc. London Math. Soc. (3) 28 (1974), 193-221. 
24. D. B. MCAlister, Some covering and embedding theorems for inverse semigroups, $J$. Austral. Math. Soc. (Ser. A) 22 (1976), 188-211.

25. D. B. MCALISTER, E-unitary inverse semigroups over semilattices, Glasgow Math. J. 19 (1978), 1-12.

26. D. B. McAlister, Regular Rees matrix semigroups and regular Dubreil-Jacotin semigroups, J. Austral. Math. Soc. (Ser. A) 31 (1981), 325-336.

27. D. B. MCAlister, Rees matrix covers for locally inverse semigroups, Trans. Amer. Math. Soc. 277 (1983), 727-738.

28. D. B. MCAlister, Rees matrix covers for regular semigroups, J. Algebra 89 (1984), 264-279.

29. D. B. MCAlister, Some covering theorems for locally inverse semigroups, J. Australian Math. Soc. (Ser. A) 39 (1985), 63-74.

30. D. B. MCAlister, Quasi-ideal embeddings and Rees matrix covers for regular semigroups, J. Algebra 152 (1992), 166-183.

31. D. B. MCALISTER and R. B. MCFADDEN, Regular semigroups with inverse transversals, Quart. J. Math. Oxford (2) 34 (1983), 459-474.

32. D. B. MCALISTER and R. B. MCFADDEN, Maximum idempotents in naturally ordered regular semigroups, Proc. Edinburgh Math. Soc. (2) 26 (1983), 213-220.

33. J. MEakin and F. PASTiJn, The structure of pseudo-semilattices. Algebra Universalis 13 (1981), 355-372.

34. D. D. Miller and A. H. Clifford, Regular $\mathscr{D}$-classes in semigroups, Trans. Amer. Math. Soc. 12 (1956), 270-280.

35. W. D. MunN, The idempotent separating congruences on a regular 0-bisimple semigroup, Proc. Edinburgh Math. Soc. (2) 15 (1967), 233-240.

36. K. S. S. NAMBOORIPAD, Structure of regular semigroups I (Mem. Amer. Math. Soc. 22 (1979), Number 224).

37. K. S. S. NAmbooripad, The natural partial order on a regular semigroup, Proc. Edinburgh Math. Soc. 23 (1980), 249-260.

38. F. Pastijn, Regular locally testable semigroups as semigroups of quasi-ideals, Acta Math. Acad. Sci. Hung. 36 (1980), 161-166.

39. M. Petrich, Inverse semigroups (John Wiley and Sons, 1984).

40. G. B. Preston, Congruences on Brandt semigroups, Math. Ann. 139 (1959), 91-94.

41. N. R. Reilly, Bisimple inverse semigroups, Proc. Glasgow Math. Assoc. 7 (1968), 101-104.

42. N. R. ReILlY and A. H. ClifFord, Bisimple inverse semigroups as semigroups of ordered triples, Canadian J. Math. 20 (1968), 25-39.

43. O. SteInfELD, Quasi-ideals in rings and semigroups (Akademiai Kiado, Budapest, 1978).

44. S. TALwar, Morita equivalence for semigroups (Preprint, University of York, 1992).

UNIVERSITY OF WALES, Bangor

SCHOOL OF MATHEMATICS

DEAN STREet

BANGOR, GWYNEDD LL57 IUT

CYMru/Wales 